\title{
Chikungunya virus impairs draining lymph node function by inhibiting HEV-mediated lymphocyte recruitment
}

\author{
Mary K. McCarthy, ${ }^{1}$ Bennett J. Davenport, ${ }^{1}$ Glennys V. Reynoso, ${ }^{2}$ Erin D. Lucas,,${ }^{1,3}$ Nicholas A. May, ${ }^{1}$ \\ Susan A. Elmore, ${ }^{4}$ Beth A. Tamburini, ${ }^{1,3}$ Heather D. Hickman, ${ }^{2}$ and Thomas E. Morrison ${ }^{1}$ \\ 'Department of Immunology and Microbiology, University of Colorado School of Medicine, Aurora, Colorado, USA. ${ }^{2}$ Viral \\ Immunity and Pathogenesis Unit, Laboratory of Clinical Microbiology and Immunology, National Institute of Allergy and \\ Infectious Diseases, NIH, Bethesda, Maryland, USA. ${ }^{3}$ Department of Medicine, University of Colorado School of Medicine, \\ Aurora, Colorado, USA. ${ }^{4}$ National Toxicology Program, National Institute of Environmental Health Sciences, Research \\ Triangle Park, North Carolina, USA.
}

Chikungunya virus (CHIKV) causes acute and chronic rheumatologic disease. Pathogenic CHIKV strains persist in joints of immunocompetent mice, while the attenuated CHIKV strain 181/25 is cleared by adaptive immunity. We analyzed the draining lymph node (dLN) to define events in lymphoid tissue that may contribute to CHIKV persistence or clearance. Acute 181/25 infection resulted in dLN enlargement and germinal center (GC) formation, while the dLN of mice infected with pathogenic CHIKV became highly disorganized and depleted of lymphocytes. Using CHIKV strains encoding ovalbumin-specific TCR epitopes, we found that lymphocyte depletion was not due to impaired lymphocyte proliferation. Instead, the accumulation of naive lymphocytes transferred from the vasculature to the dLN was reduced, which was associated with fewer high endothelial venule cells and decreased CCL21 production. Following NP-OVA immunization, NP-specific CC B cells in the dLN were decreased during pathogenic, but not attenuated, CHIKV infection. Our data suggest that pathogenic, persistent strains of CHIKV disable the development of adaptive immune responses within the dLN.

Conflict of interest: The authors have declared that no conflict of interest exists.

Submitted: March 15, 2018

Accepted: June 6, 2018

Published: July 12, 2018

\section{Reference information:}

JCI Insight. 2018;3(13):e121100.

https://doi.org/10.1172/jci.

insight.121100.

\section{Introduction}

Chikungunya virus (CHIKV) is a mosquito-transmitted RNA virus that reemerged in the last decade to cause outbreaks of rheumatologic disease on an unprecedented scale (1-5), including outbreaks in the Americas that affected millions (6). CHIKV infection generally presents with symptoms such as fever, severe joint pain, muscle aches, and rash, which subside in 7-10 days. However, up to two-thirds of those infected experience incapacitating arthralgia, joint inflammation, and tenosynovitis that persist for months or years after the acute phase (7-15).

In humans, chronic disease is associated with detectable CHIKV RNA and antigen in musculoskeletal tissue $(16,17)$, suggesting that host immune responses fail to clear CHIKV from some tissues. In experimentally infected macaques, joints, muscles, lymphoid organs, and liver harbor infectious CHIKV or CHIKV RNA for months $(18,19)$. Wild-type (WT) C57BL/6 mice infected with CHIKV develop chronic synovitis (20), and CHIKV RNA and infectious virus can be detected in joint-associated tissue for months (20-23). The severity of chronic joint disease is increased in aged WT mice with persistent CHIKV infection, and is associated with defects in adaptive immunity (22). Moreover, Rag $1^{1^{-1}}$ mice persistently infected with CHIKV develop more severe chronic inflammation in joint-associated tissue than WT mice (20), suggesting that adaptive immune responses protect against chronic musculoskeletal disease.

Arboviruses are deposited into the skin when a virus-infected mosquito probes for a blood meal (24). Viral antigen, nucleic acid, and live virus are transported in the lymph or via skin-resident Langerhans cells or other DCs to secondary lymphoid tissue (25-27), which is essential for immunological surveillance and the development of adaptive immune responses (28). Given the inability of adaptive immune responses to clear pathogenic CHIKV infection, we investigated responses in the draining lymph node (dLN), the first lymphoid tissue to encounter and respond to the infection. To facilitate our analyses, we performed comparative studies 
with genetically similar CHIKV strains that differ in virulence and by only 5 amino acids across the genome: a pathogenic clinical isolate AF15561 and the derivative attenuated strain 181/25 (29, 30). Whereas AF15561 establishes a chronic infection, $181 / 25$ is cleared from musculoskeletal tissue of immunocompetent mice in a manner dependent on adaptive immunity (31).

In this study, we find that infection with pathogenic strains of CHIKV, but not the attenuated 181/25 strain, causes lymphocyte depletion and disorganization in the dLN. Only infection with CHIKV 181/25 resulted in increased $\mathrm{B}$ and $\mathrm{T}$ cell numbers in the $\mathrm{dLN}$ and the development of germinal centers (GCs). High endothelial venule (HEV) expansion and CCL21 production were impaired during pathogenic $\mathrm{CHIKV}$ infection. Consistent with these observations, the accumulation of naive lymphocytes transferred from the vasculature to the dLN was reduced. Furthermore, pathogenic CHIKV infection impaired the dLN response to immunization. Thus, early defects in $\mathrm{dLN}$ responses during pathogenic CHIKV infection impair the development of adaptive immune responses and likely contribute to viral persistence.

\section{Results}

Pathogenic CHIKV infection disrupts the architecture of the dLN. CHIKV strain AF15561 establishes a persistent infection in musculoskeletal tissue, while the derivative 181/25 strain, which differs by 5 amino acids, is cleared by adaptive immune responses (31). Secondary lymphoid organs (SLOs) are critical for the generation of antiviral adaptive immune responses and control of viral infection (22, 26-28, 32-35). To assess early immune responses at the onset of what will become an acutely cleared $(181 / 25)$ or persistent (AF15561) CHIKV infection, we focused on the popliteal dLN, the first lymphoid organ to encounter antigen following inoculation of CHIKV in the footpad. H\&E staining of fixed dLN sections from mockinfected mice showed LNs with follicles but no GCs, and normal paracortical regions (Figure 1A). By 3 days postinfection (dpi), the dLN of 181/25-infected mice were enlarged, and beginning at 7 dpi had expanded paracortexes, increased follicles, and the presence of GCs, hallmarks of the adaptive immune response to infection (36) (Figure 1, A and B). In contrast, the dLN of AF15561-infected mice lacked GC development. Instead, the dLN became depleted of lymphocytes and mildly fibrotic by 7 dpi. At 10 dpi, dLNs from AF15561-infected mice displayed marked fibrosis and lymphocyte depletion, which decreased by $14 \mathrm{dpi}$ (Figure 1, A-C). These histopathological changes were unique to the dLN and were not present in other peripheral LNs examined (left inguinal) or in the spleen (data not shown). These data suggest that pathogenic, persistent $\mathrm{CHIKV}$ infection disrupts nodal expansion after infection, while acutely cleared CHIKV infection generates robust dLN enlargement and a GC response.

Decreased lymphocyte numbers and altered lymphocyte subset localization in the $d L N$ during pathogenic CHIKV infection. LN hypertrophy during early immune responses is largely mediated by increased numbers of lymphocytes (37-43). Therefore, we evaluated lymphocyte populations in the dLN during the first week of infection. During 181/25 infection, total cell numbers in the dLN were increased over mock-infected mice at 3 dpi and continued to increase through 10 dpi (Figure 2A). The majority of cells contributing to the increase were $\mathrm{B}$ cells, although $\mathrm{CD} 4^{+} \mathrm{T}$ cells and $\mathrm{CD} 8^{+} \mathrm{T}$ cells also contributed (Figure 2, $\mathrm{B}-\mathrm{D})$. In contrast, during AF15561 infection, total cell numbers in the dLN were higher than in mock- or 181/25-infected mice at $1 \mathrm{dpi}$, after which we observed a marked decrease in cellularity (Figure 2A). The decreased cellularity in the dLN of AF15561-infected mice was not due to the absence of a singular lymphocyte subset, but a decrease in the number of all lymphocyte subsets examined: B cells, CD4 ${ }^{+} \mathrm{T}$ cells, CD8 ${ }^{+} \mathrm{T}$ cells, NK1.1 $1^{+}$cells, and NK1.1 ${ }^{+} \mathrm{CD}^{+} \mathrm{NKT}$ cells (Figure 2, B-D and Supplemental Figure 1, A and B; supplemental material available online with this article; https://doi.org/10.1172/jci. insight.121100DS1). Consistent with the lymphocyte depletion and lack of GC development observed during AF15561 infection, there was a decrease in the percentage and number of T follicular helper (Tfh) cells in the dLN at 7 dpi (Supplemental Figure 1, C-E). We did not observe an increased percentage of apoptotic (annexin $\mathrm{V}^{+}$) or dead (annexin $\mathrm{V}^{+}$AquaVI ${ }^{+}$) B cells, $\mathrm{CD}^{+} \mathrm{T}$ cells, or $\mathrm{CD}^{+} \mathrm{T}$ cells in the dLN of AF15561-infected mice at 5 dpi (Supplemental Figure 2, A-E), suggesting that lymphocyte depletion during pathogenic CHIKV infection was not due to increased lymphocyte death or apoptosis. In support of this, fibroblastic reticular cells (FRCs), which provide a 3-dimensional support network for lymphocyte migration and produce cytokines important for lymphocyte survival (such as IL-7), expanded similarly within the first week after 181/25 and AF15561 infection (Supplemental Figure 3, A-C).

To investigate whether there were differences in lymphocyte subset localization prior to the development of fibrosis, we analyzed ERTR $-7^{+}$stromal cells, B220 $0^{+}$B cells, and CD8 ${ }^{+} \mathrm{T}$ cells in frozen dLN sections by 
A

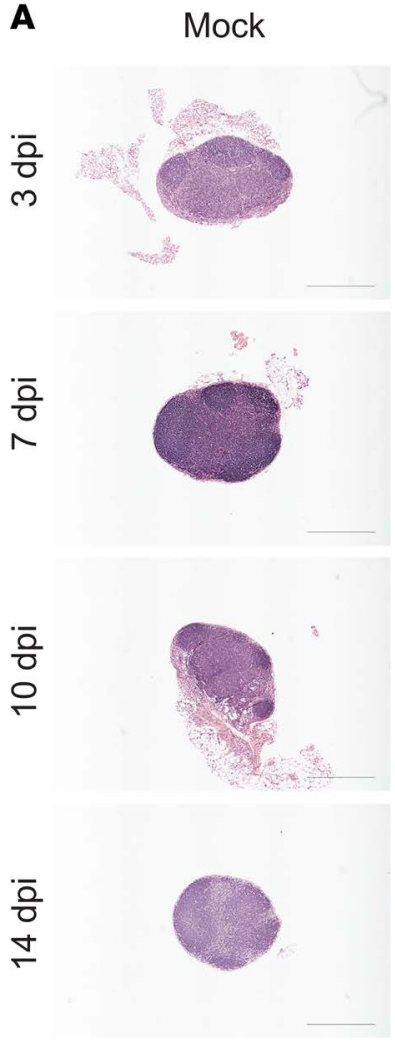

$181 / 25$

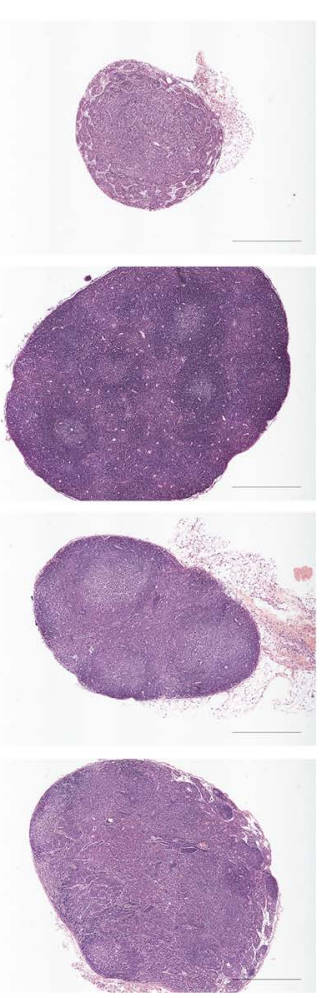

AF15561

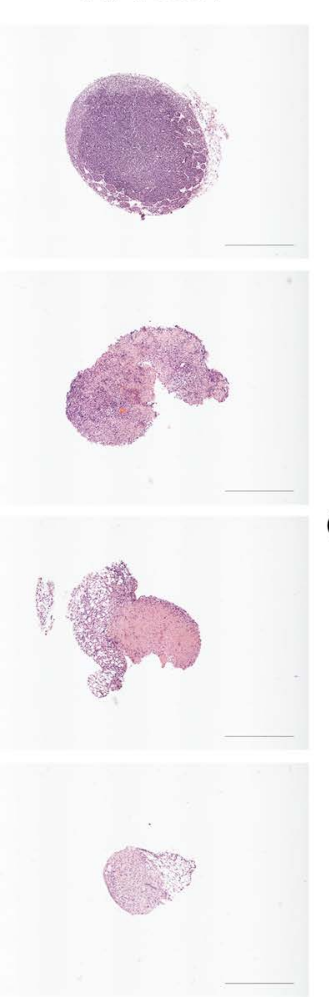

B Increased Development of Germinal Centers

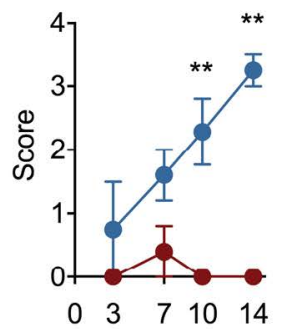

Days Post Inoculation

$181 / 25$ AF15561 Decreased Lymphocytes

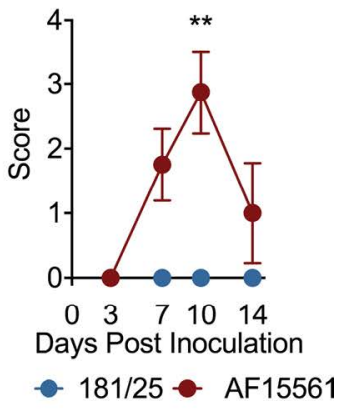

Figure 1. Pathogenic CHIKV infection disrupts the architecture of the draining lymph node. WT C57BL/ 6 mice were mock infected or infected with $10^{3}$ PFU 181/25 or AF15561 via the left rear footpad and the dLN was excised at the indicated time points (3-8 mice per group). (A) H\&E-stained sections were prepared from paraffin-embedded draining popliteal lymph nodes. Scale bars: $500 \mu \mathrm{m}$. Sections were scored for the degree of (B) GC development, and (C) fibrosis and decreased lymphocytes at the indicated time points. Data represent the mean \pm SEM of 3-4 (3 dpi), 5-6 (7 dpi), 7-8 (10 dpi), or 4-5 (14 dpi) mice per group. ${ }^{* *} P<0.01$; Kruskal-Wallis test with Dunn's multiple-comparisons test.

confocal microscopy. These analyses revealed that the failed accumulation of lymphocytes in the dLN after AF15561 infection was accompanied by marked alterations in lymphocyte localization. At 1 dpi, the general organization of the dLN appeared similar in mock-, 181/25-, and AF15561-infected mice, with well-defined follicles subjacent to the node's capsule and a clearly defined B cell/T cell border (Figure 2E). This remained the case for 181/25 dLNs through at least 5 dpi. However, the dLNs of AF15561-infected mice displayed dramatic follicular disruption at 3 and $5 \mathrm{dpi}$, with central, paracortical relocalization of B cells. In addition, $\mathrm{CD}^{+} \mathrm{T}$ cells were positioned more diffusely throughout the $\mathrm{dLN}$, with an almost complete loss of the $\mathrm{B}$ cell/T cell border by 3 dpi (Figure 2E). Together, these results suggest that pathogenic, persistent CHIKV infection leads to lymphocyte depletion and mislocalization in the dLN.

Disruption of $d L N$ architecture and lymphocyte depletion is a common feature of pathogenic CHIKV infection and depends on 2 residues in the E2 glycoprotein. The E2 glycoprotein of the acutely cleared CHIKV strain $181 / 25$ contains 2 mutations, T12I and G82R, that promote viral clearance from murine joint tissues (31). Reversion of either residue in $181 / 25$ at E2 position 12 or 82 to the WT residue resulted in decreased total cell numbers in the dLN compared with 181/25 infection (Figure 3A). However, reversion of both residues $\left(181 / 25^{\mathrm{E} 2}\right.$ I12T $\left.{ }^{\mathrm{R} 82 \mathrm{G}}\right)$ was required for dLN cell numbers equivalent to those detected in dLNs of AF15561-infected mice (Figure 3A). These results suggest that the threonine and glycine at E2 positions 12 and 82, respectively, contribute to lymphocyte depletion in the dLN observed after AF15561 infection.

To determine whether the decreased cellularity and lack of GC development in the dLN was a unique feature of infection with CHIKV AF15561, an Asian genotype strain, or a common feature of infection with pathogenic, persistent CHIKV strains, we analyzed the dLN in mice infected with CHIKV SL15649, an East, Central, South African (ECSA) genotype strain (44). Like AF15561, SL15649 also contains $\mathrm{a} T$ and $\mathrm{G}$ at $\mathrm{E} 2$ position 12 and 82, respectively. Following SL15649 infection, total cell numbers in the dLN did not increase by 5 dpi (Figure 3B). At 10 dpi, the dLNs of SL15649-infected mice 

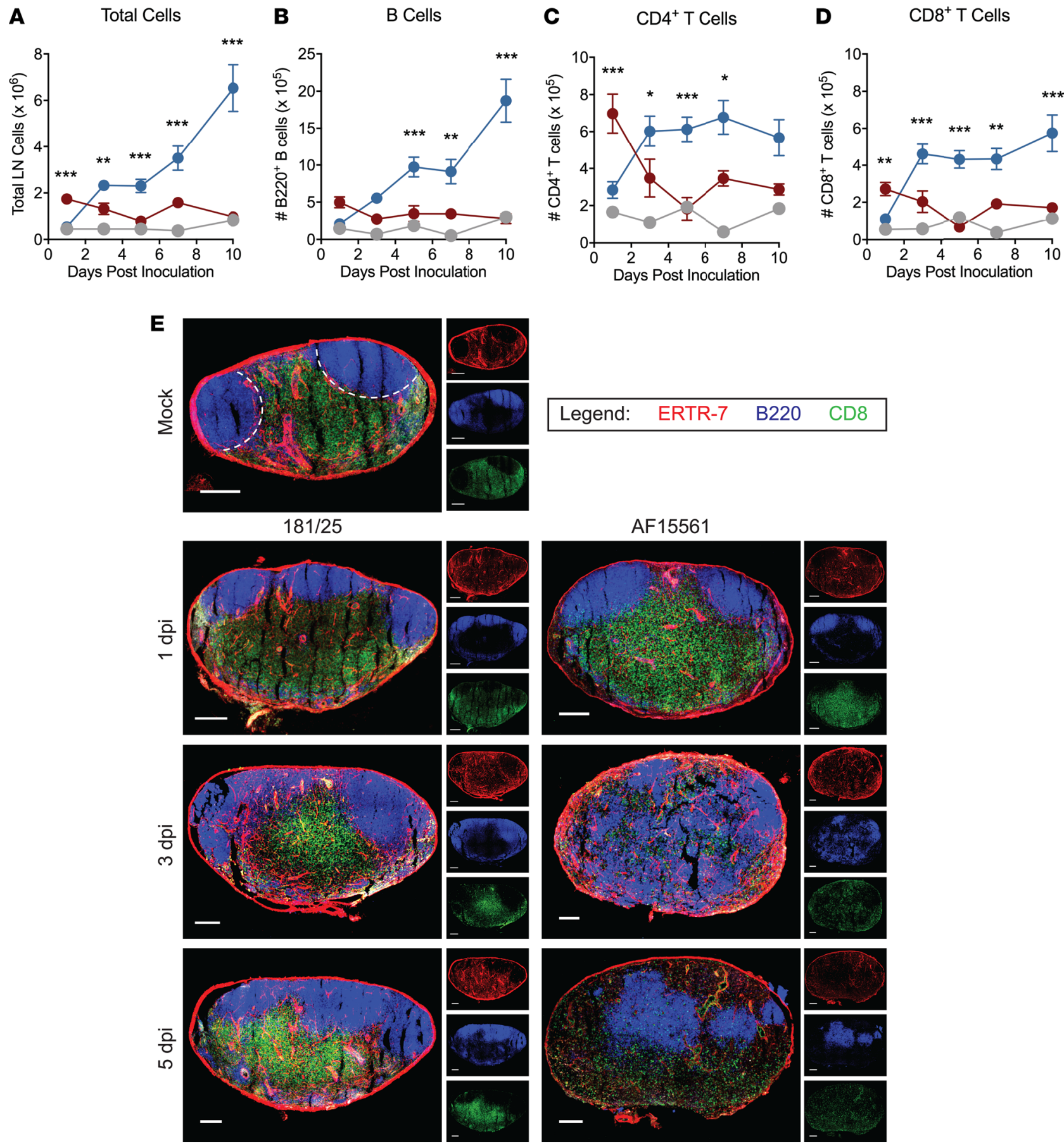

Figure 2. Decreased lymphocyte numbers and altered lymphocyte subset localization in the dLN after pathogenic CHIKV infection. (A) Total cells in dLN. Mock (gray), 181/25 (blue), AF15561 (red). Total numbers of (B) B220+ cells, (C) CD4+ T cells, and (D) CD8+ T cells in the dLN. Data are derived from 2-3 independent experiments per time point. Each data point represents the mean \pm SEM of 9-11 (1 dpi), 6-10 (3 dpi), 6-8 (5 dpi), 4-5 (7 dpi), or 7-8 (10 dpi) mice per group. ${ }^{*} P<0.05$, ${ }^{* *} P<0.01$, ${ }^{* *} P<0.001$; two-way ANOVA with Bonferroni's post hoc test. Statistical significance is only displayed for comparison of $181 / 25$ and AF15561 groups. (E) Confocal micrographs showing ERTR-7 $7^{+}$stromal cells (red), B220+ B cells (blue), and CD8 ${ }^{+}$T cells (green) in the dLN at the indicated time points. White dashed line in mock LN outlines B cell follicles. Images are representative of 3-6 dLNs per group per time point. Scale bars: $200 \mu \mathrm{m}$.

displayed marked lymphocyte depletion (Figure 3C) and lacked GC development (Figure 3D). These data suggest that disruption of early dLN responses is a common feature of infection with pathogenic, persistent CHIKV strains.

Lymphocyte proliferation in the $d L N$ is similar during attenuated and pathogenic CHIKV infection. We observed fewer lymphocytes in the dLN after pathogenic, persistent CHIKV infection (Figure 2, A-D and 
A
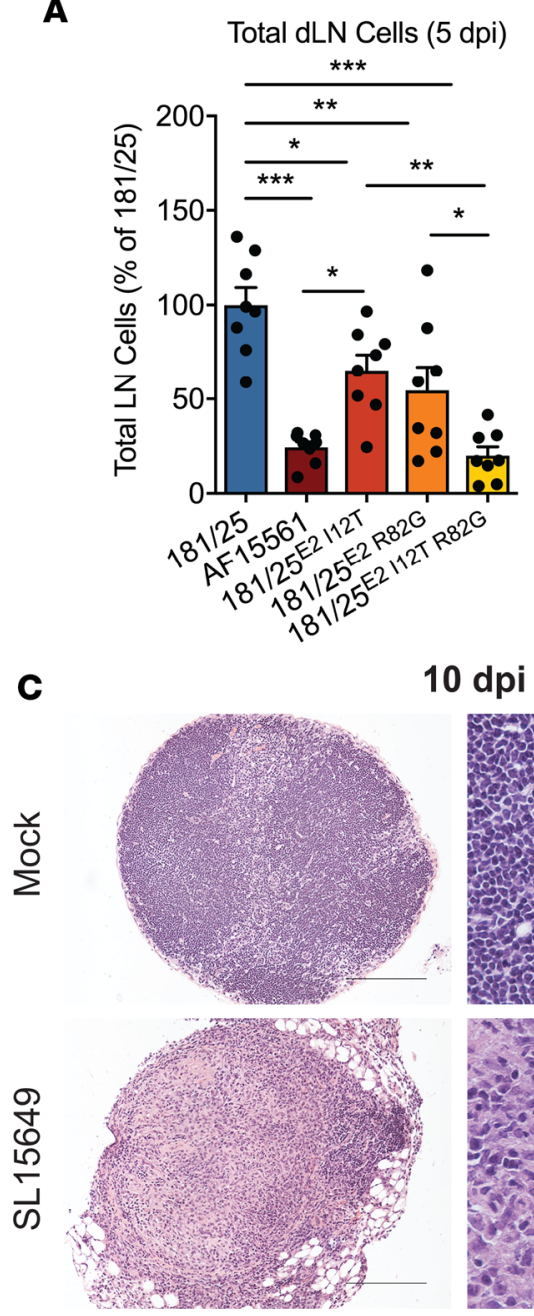

$10 \mathrm{dpi}$

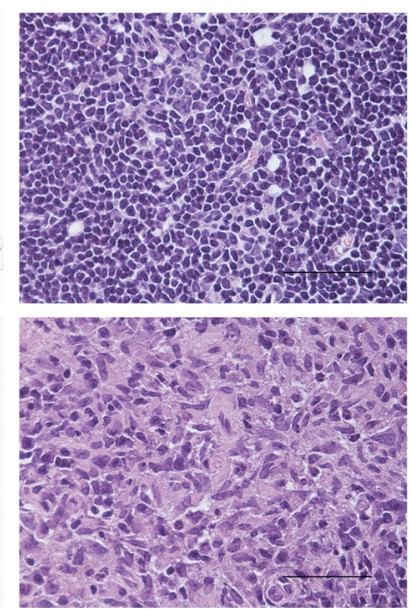

B Total dLN Cells (5 dpi)

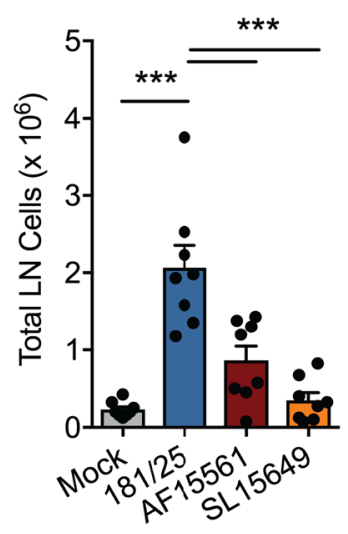

Figure 3. Disruption of $d \mathrm{LN}$ architecture and lymphocyte depletion is a common feature of pathogenic CHIKV infection and depends on 2 residues in the E2 glycoprotein. (A) Total cells in the dLN at 5 dpi of mice infected with CHIKV 181/25,

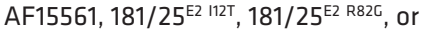
$181 / 25^{\text {E2 }}$ 112T R82C (displayed as a percentage of cells in 181/25-infected mice). (B) Total cells in the dLN at 5 dpi of mice infected with CHIKV 181/25, AF15561, or SL15649. (C) At 10 dpi, H\&E-stained sections were prepared from the dLN. Scale bars: $200 \mu \mathrm{m}$ (left) or $50 \mu \mathrm{m}$ (right). (D) Sections were scored for the degree of CC development. 181/25 and AF15561 data points in part $\mathbf{B}$ are the same as those displayed in Figure 1. Data are derived from 2 independent experiments with 5-8 mice per group. ${ }^{*} P<0.05,{ }^{*} P$ $<0.01$, ${ }^{* *} P<0.001$; one-way ANOVA with Tukey's multiple comparisons test (A and B) or a Kruskal-Wallis test with Dunn's multiple-comparisons test (D).

Figure 3B). Because lymphocyte proliferation contributes to LN hypertrophy, we hypothesized that there may be a defect in lymphocyte proliferation in the dLN after pathogenic, persistent CHIKV infection. To evaluate the proliferation of bulk and CHIKV-specific T cells, we engineered recombinant CHIKV strains containing the $\mathrm{H}-2 \mathrm{~K}^{\mathrm{b}}$-restricted ovalbumin $\mathrm{OVA}_{257-264}$ epitope (SIINFEKL) and the I-A $\mathrm{A} / \mathrm{I}-\mathrm{A}^{\mathrm{b}}-$ restricted OVA $_{323-339}$ epitope (ISQAVHAAHAEINEAGR) in frame with the CHIKV structural polyprotein in the 181/25 (181/25.OVA) or AF15561 (AF15561.OVA) genome (Supplemental Figure 4A), using a strategy that we previously used to evaluate $\mathrm{T}$ cell responses during Ross River virus infection (45). 181/25.OVA and AF15561.OVA grew similarly in BHK-21 cells (Supplemental Figure 4B). Similar to infection with the parental viruses, we detected more cells in the dLN of 181/25.OVA-infected mice than AF15561. OVA-infected mice at both 3 and 5 dpi (Supplemental Figure 4C). Importantly, 181/25. OVA was cleared from joint tissue of WT mice by $28 \mathrm{dpi}$, but persisted in B cell-deficient ( $\mu \mathrm{MT})$ mice, as observed with WT 181/25 (31). AF15561.OVA persisted in both WT and $\mu$ MT mice (Supplemental Figure 4D). WT mice generated a robust $\mathrm{CD} 8^{+} \mathrm{T}$ cell response to the $\mathrm{OVA}_{257-264}$ epitope in the spleen after infection with either 181/25.OVA or AF15561.OVA, but not the parental viruses (Figure 4, A and B), demonstrating that the recombinant OVA virus strains produce a specific response to the $\mathrm{OVA}_{257-264}$ epitope.

Following infection with either 181/25.OVA or AF15561.OVA, there were little to no differences in Ki67 expression in total $\mathrm{CD} 44^{+} \mathrm{CD} 4^{+}$or $\mathrm{CD} 44^{+} \mathrm{CD} 8^{+} \mathrm{T}$ cells, or total B cells in the dLN at 5 dpi (Supplemental Figure 4, E-G). In addition, the percentage of SIINFEKL tetramer ${ }^{+} \mathrm{CD} 8^{+} \mathrm{T}$ cells that were Ki67 ${ }^{+}$within the dLN during 181/25.OVA and AF15561.OVA infection was comparable (Figure 4, C-E), although there were fewer tetramer ${ }^{+} \mathrm{CD} 8^{+} \mathrm{T}$ cells in the $\mathrm{dLN}$ of AF15561.OVA-infected mice (Figure 4D). To evaluate proliferation of OVA-specific $\mathrm{CD} 4^{+} \mathrm{T}$ cells, we adoptively transferred enriched $\mathrm{CD} 4^{+} \mathrm{T}$ cells from CD45.1 $1^{+}$OT-II mice prior to infection with 181/25.OVA or AF15561.OVA and analyzed the dLN at 
A Spleen, 7 dpi - gated on $B 220-C D 3^{+} C D 8^{+}$cells

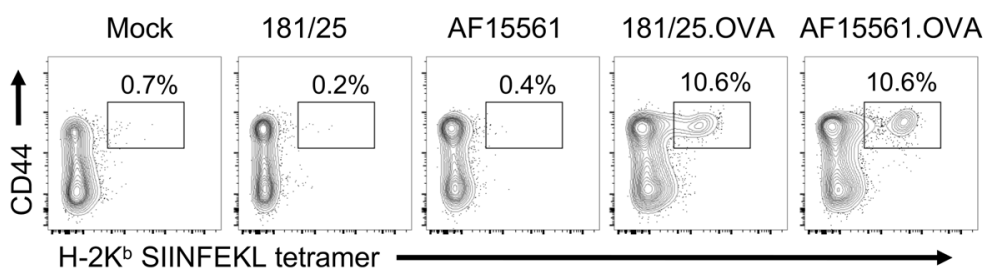

C dLN, 5 dpi - gated on $B 220-C D 3^{+} C D 8^{+}$cells
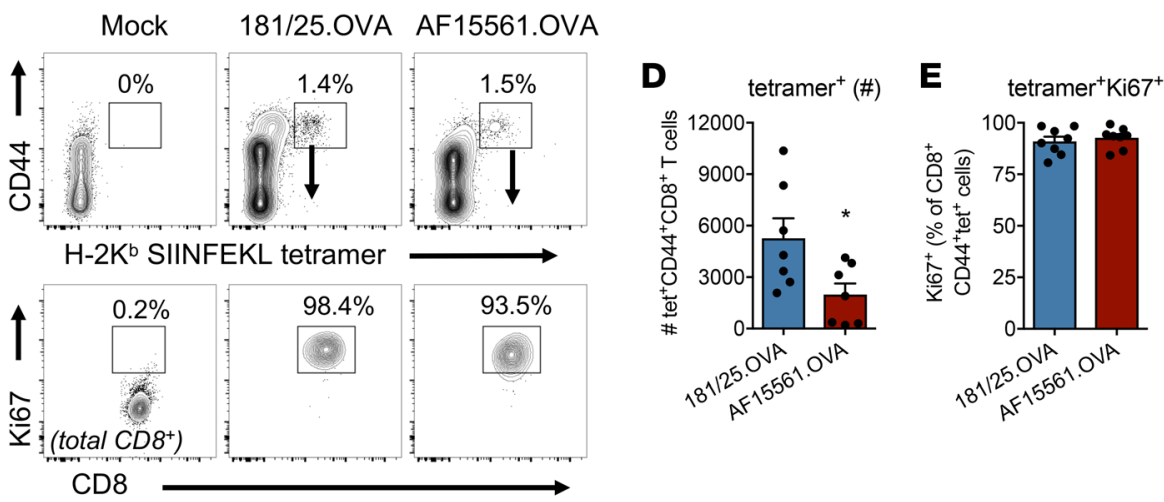

$\mathbf{F}$
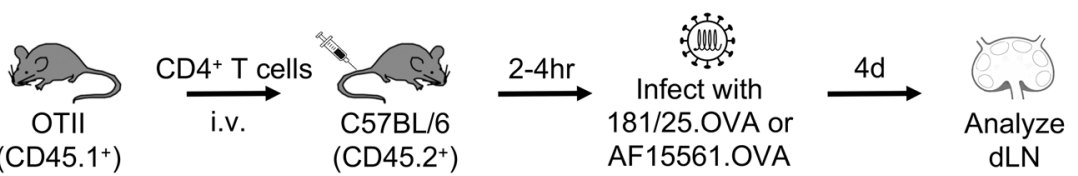

$\mathbf{G}$

dLN, 4 dpi - gated on CD45.1+CD4+ cells
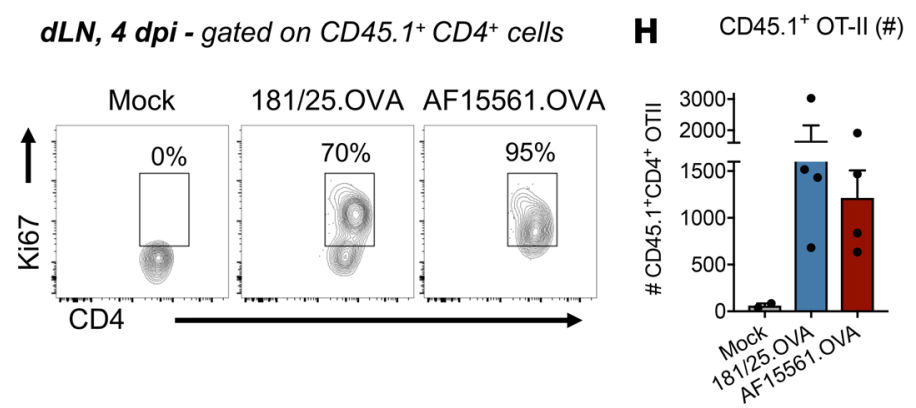

B

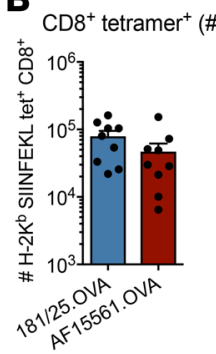

Figure 4. Lymphocyte proliferation in the dLN is similar during acutely cleared and pathogenic CHIKV infection. (A) Representative FACS plots and (B) number of SIINFEKL tetramer+ $\mathrm{CD}^{+}$ T cells in the spleen of WT C57BL/ 6 mice ( $n=9$ per group) at $7 \mathrm{dpi}$. (C-E) WT C57BL/ 6 mice ( $n=8$ per group) were mock infected or infected with $10^{3} \mathrm{PFU}$ 181/25.0VA or AF15561.0VA and the dLN was excised at 5 dpi. (C) Frequency of CD8 ${ }^{+}$SIINFEKL tetramer ${ }^{+}$cells (top panels) and $\mathrm{Ki} 67^{+}$of total $\mathrm{CD} 8^{+}$cells or tetramer+ cells (bottom panels). (D) Number of SIINFEKL tetramer ${ }^{+}$CD8 ${ }^{+} \mathrm{T}$ cells. (E) Percentage of CD8 ${ }^{+}$SIINFEKL tetramer+ cells expressing Ki67. Data are combined from 2 independent experiments. (F) Enriched $\mathrm{CD} 4^{+} \mathrm{T}$ cells $\left(10^{5}\right)$ from CD45.1 $1^{+}$OT-II mice were transferred i.v. into CD45. $2^{+}$recipients $(n=4$ per group) 4 hours prior to inoculation with $10^{3}$ PFU 181/25.OVA or AF15561.0VA and the dLN was collected at 4 dpi. (C) FACS plots showing Ki67+ OT-II cells. (H) Number of transferred OT-II cells per dLN. (I) Percentage of Ki67+ OT-II cells. Data are representative of 2 independent experiments. Each bar represents the mean \pm SEM. ${ }^{*} P<0.05$, ${ }^{* * *} P<0.001$; MannWhitney test (E and $\mathbf{F}$ ), or 1-way ANOVA with Tukey's multiple-comparisons test (A-C, I, and J).

4 dpi (Figure 4F). By this time point, the majority of the transferred OT-II cells were $\mathrm{Ki}^{+} 7^{+}$in the $\mathrm{dLN}$ of infected mice (Figure 4, G-I). These results suggest that the decreased cellularity of the dLN during pathogenic, persistent CHIKV infection is not due to impaired B or T lymphocyte proliferation within the dLN.

Reduced HEV cell expansion in the $d L N$ after pathogenic CHIKV infection. Naive L-selectin ${ }^{\text {hi }}$ lymphocytes enter the dLN from the blood through specialized blood vessels (HEVs) (46). HEV cells can be distinguished from total CD45-CD31 ${ }^{+}$endothelial cells based on expression of MECA-79, also known as peripheral node addressin (PNAd), a component of the L-selectin adhesion cascade $(42,47,48)$. We first analyzed HEV cells by flow cytometry to determine whether there was a difference in the expansion of HEVs, which occurs as the dLN enlarges after infection. HEV cells increased in number in both 181/25- and AF15561-infected nodes on both days 5 and $7 \mathrm{dpi}$, although to a much lesser extent after infection with AF15561 (Figure 5, A and B). Expression levels of adhesion molecules PNAd or ICAM-1 on HEV cells did not differ between 181/25 and AF15561 infection (Supplemental Figure 5, A and B), suggesting that AF15561 does not alter expression of these molecules involved in lymphocyte adhesion and extravasation to the LN. Decreased lymphocyte numbers were observed in dLNs of AF15561-infected mice as early as 3 dpi (Figure 2, A-D), prior to measurable expansion of HEV endothelial cells by flow cytometry. Therefore, we analyzed HEVs by confocal microscopy of frozen LN sections at 3,5, and 7 dpi to determine whether there were any overt differences in HEV area or morphology between the 2 infections (Figure 5, C and D). HEVs were localized in 
closer proximity to the dLN capsule after AF15561 infection compared with 181/25 infection (Supplemental Figure 5C), which is associated with the lack of follicular expansion and B cell mislocalization observed in dLNs of AF15561-infected mice (Figure 2). The number of HEVs per section and the area per HEV were reduced in the dLNs of AF15561-infected mice (Supplemental Figure 5, D-F), resulting in a significantly lower total HEV area per section at 3, 5, and 7 dpi (Figure 5E). These data suggest that pathogenic CHIKV infection impairs expansion of HEV cells in the dLN.

The homeostatic chemokine CCL21 is expressed by HEV endothelial cells and mediates naive T cell extravasation through HEVs into LNs (46). In addition to decreased total HEV area for $\mathrm{T}$ cell entry, we hypothesized that alterations in CCL21 production during AF15561 infection could contribute to decreased T cell entry. While CCL21 protein levels were significantly increased in the dLNs of 181/25-infected mice at both 1 and 3 dpi, CCL21 did not increase after AF15561 infection (Figure 5F). Consistent with impaired CCL21 expression, confocal microscopy analysis showed alterations in CD ${ }^{+}$ $\mathrm{T}$ cell localization in the $\mathrm{dLN}$ of AF15561-infected mice. CD8 ${ }^{+} \mathrm{T}$ cells were localized in close proximity to or on the luminal surface of PNAd ${ }^{+} \mathrm{HEVs}$ after AF15561, but not 181/25 infection (Figure 5, D and G). These data suggest that defects in expression of the homeostatic chemokine CCL21 are apparent prior to measurable HEV expansion, and this is associated with alterations in localization of $\mathrm{CD} 8^{+} \mathrm{T}$ cells at the HEV during pathogenic CHIKV infection.

Accumulation of naive lymphocytes in the $d L N$ is impaired during pathogenic CHIKV infection. The failure to upregulate CCL21, decreased HEV area, and concentration of CD8 ${ }^{+} \mathrm{T}$ cells on the luminal surface of HEVs early after AF15561 infection suggested that lymphocyte recruitment into the LN through the HEVs might be impaired in AF15561-infected dLNs. Thus, we assessed whether the accumulation of naive lymphocytes in SLOs was affected after pathogenic, persistent CHIKV infection. CFSE-labeled naive splenocytes were transferred intravenously (i.v.) into mock-, 181/25-, or AF15561-footpad-infected mice at $4.5 \mathrm{dpi}$ and the number of transferred cells in the spleen, left iliac LN, left inguinal LN, and left popliteal dLN was determined by flow cytometry 12 hours after transfer (Figure 6, A and B). The total number of transferred cells in the spleen, left iliac LN, or left inguinal LN was similar in $181 / 25-$ or AF15561-infected mice (Figure 6C). Remarkably, despite equivalent accumulation of transferred cells in all other SLOs in infected mice, we failed to detect enhanced recruitment of transferred cells into the dLN of AF15561-infected mice (Figure 6, B and C). Additionally, defective lymphocyte recruitment was present as early as $3 \mathrm{dpi}$ (Supplemental Figure 6). These data suggest that lymphocyte entry via HEVs is impaired uniquely in the dLN following pathogenic, persistent CHIKV infection.

Stromal cells in the dLN are not targets of productive CHIKV infection. In prior studies, we found that the pathogenic, persistent CHIKV strain AF15561 and the acutely cleared 181/25 strain replicate to similar titers at the site of inoculation during the first week of infection (49). Infectious virus is present in homogenized dLNs of WT mice infected with pathogenic CHIKV strains and 181/25, although the levels are reduced in 181/25-infected mice $(50,51)$. Moreover, increased viral burdens are detected in lymphoid tissue of mice infected with viruses that contain a glycine at $\mathrm{E} 2$ position 82 , which is required for disruption of early responses in the dLN (Figure 3A) (49). However, whether this detected virus represents passive or active transport from the site of infection to the dLN or the productive infection of LN-resident cells has not been defined. We hypothesized that pathogenic, persistent CHIKV strains infect one or more cell populations in the dLN, contributing to disorganization of the dLN, disrupted chemokine production, the loss of HEV endothelial cells, and ultimately the decreased lymphocyte recruitment. To investigate this, we engineered a recombinant version of AF15561 expressing mKate (AF15561.mKate), permitting identification of productively infected cells in vivo by flow cytometry. The AF15561.mKate virus was not attenuated in vitro compared with the parental virus, and the percentage of $\mathrm{mKate}^{+}$cells in culture increased over time (Figure 7, A and B). In WT mice infected with AF15561.mKate, but not WT AF15561, we detected mKate ${ }^{+}$cells in the inoculated foot at 24 hours postinfection (hpi) (Figure 7C). However, $\mathrm{mKate}^{+}$cells were undetectable within the CD45 ${ }^{+}$and CD45 populations of dLNs (Figure 7C). To further explore the possibility that LN cells are productively infected, we performed similar analyses in Ifnar $1^{-/-}$mice, in which CHIKV causes a severe disseminated infection due to the absence of type I IFN signaling (50-52). A small population of $\mathrm{mKate}^{+}$cells was present in the dLNs of Ifnar $1^{-/-}$mice, representing approximately $0.1 \%-0.2 \%$ of all CD $45^{-}$cells (Figure $7 \mathrm{C}$ ). Collectively, these data suggest that cells in the dLN are not sites of robust viral replication during AF15561 infection of WT mice, and that only a small portion of stromal cells in the dLN become infected even when key components of the type I IFN pathway are absent. Thus, differential productive infection of resident cells of the dLN by $181 / 25$ 
A

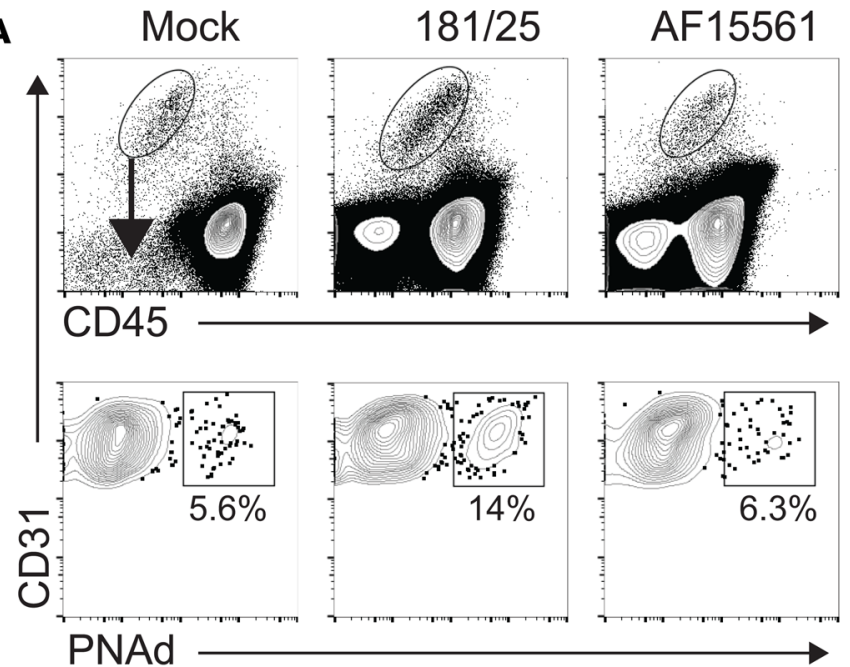

B

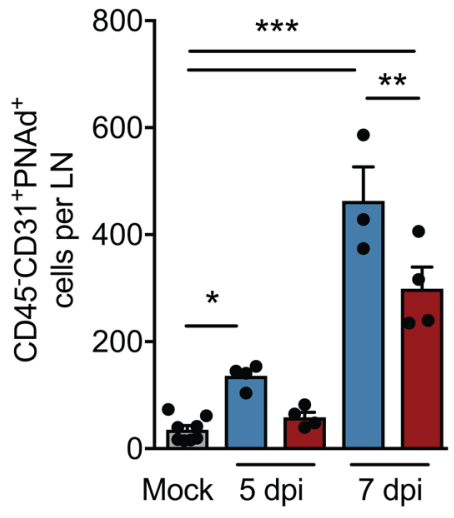

Mock $\square$ 181/25
AF15561
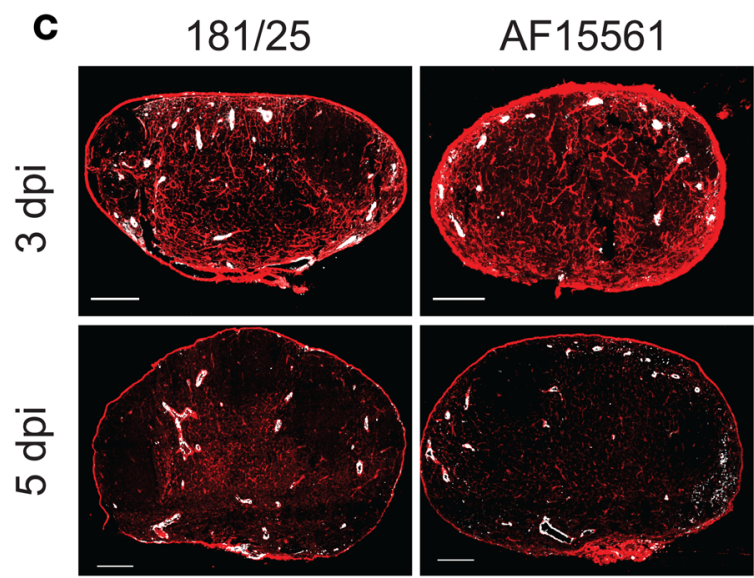

D

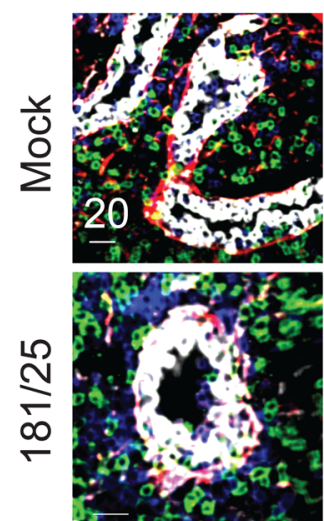

5 dpi
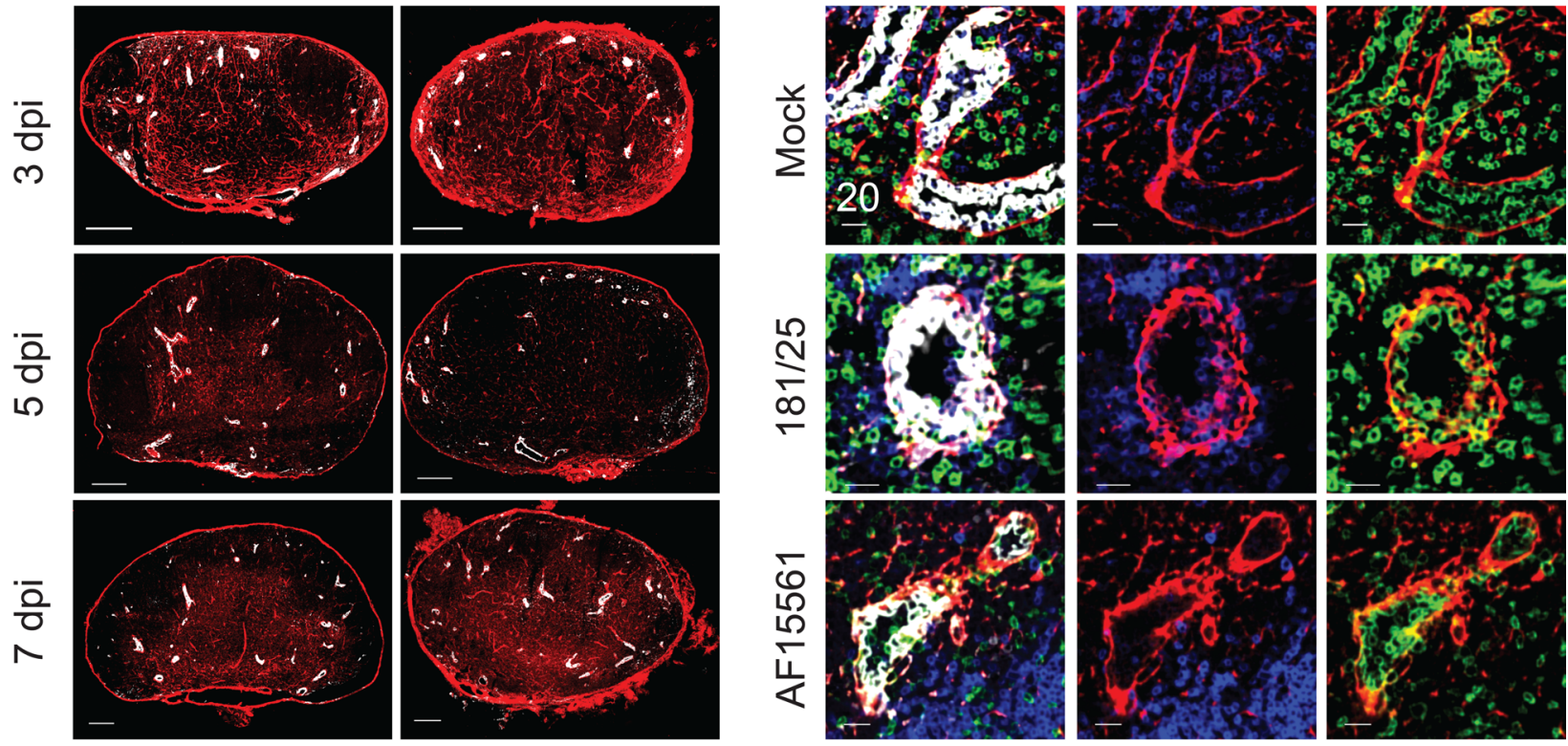

ERTR-7 PNAd (white)

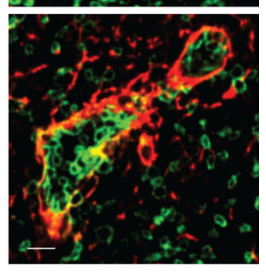

B220

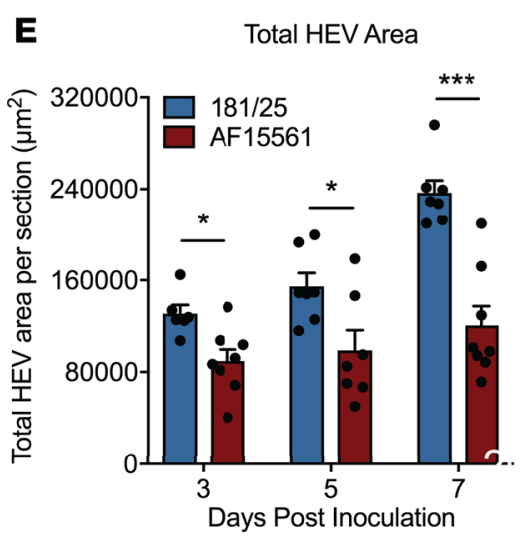

$\mathbf{F}$

CCL21

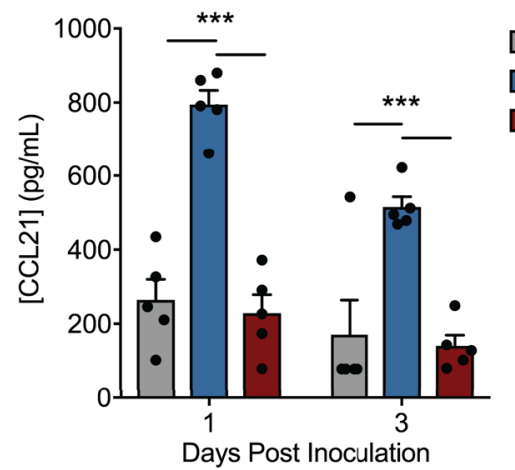

G $\quad \mathrm{CD}^{+} \mathrm{T}$ cell concentration in HEVs (5 dpi)
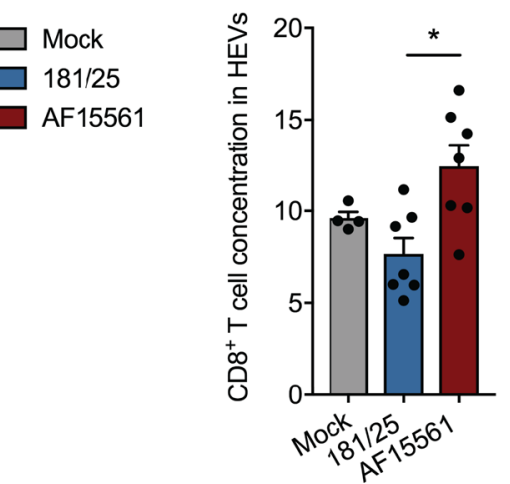

Figure 5. Reduced HEV cell expansion in the dLN during pathogenic CHIKV infection. WT C57BL/6 mice were inoculated in both rear footpads with $10^{3}$ PFU of CHIKV 181/25 or AF15561. (A) At 5 or 7 dpi, popliteal LNs were collected and analyzed by flow cytometry to identify stromal cell populations. Gating strategy to identify HEVs based on CD45-CD31+ (top panels) and PNAd ${ }^{+}$cells (bottom panels). (B) Number of CD45-CD31+PNAd ${ }^{+}$HEVs per $^{-}$ LN. Data are representative of 3 independent experiments and each bar represents the mean \pm SEM of 3-8 mice per group. (C) Confocal micrographs 
showing ERTR-7 ${ }^{+}$stromal cells (red) and PNAd ${ }^{+}$HEVs (white) in the dLN at the indicated time points. Images are representative of 3-6 dLNs per

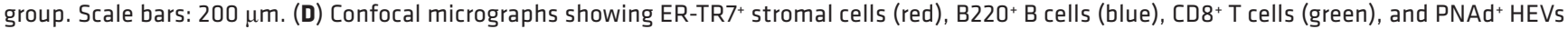
(white) in the dLN at $5 \mathrm{dpi}$. Scale bars: $20 \mu \mathrm{m}$. (D) Total HEV area per section ( $n=3-6$ individual mice per group, 1-2 sections analyzed per mouse). (F) CCL21 protein levels in the dLN were measured by ELISA at the indicated time points ( $n=5$ per group). (G) Concentration coefficient for CD8 fluorescence signal in HEV lumen. ${ }^{*} P<0.05,{ }^{* *} P<0.01,{ }^{* *} P<0.001$; one-way ANOVA with Tukey's multiple-comparisons test (B), Mann-Whitney test (E and $\mathbf{G})$, or 2-way ANOVA with Bonferroni's multiple-comparisons test (F).

and AF15561 is unlikely to fully explain the phenotypic and functional differences observed in the dLN after infection with the 2 virus strains.

Pathogenic, but not acutely cleared, CHIKV infection, impairs the dLN response to immunization. The reduced number of $\mathrm{T}$ and $\mathrm{B}$ cells within $\mathrm{dLN}$ s after AF15561 infection and disrupted T/B border (where $\mathrm{T}$ cell-dependent B cells become activated), suggested the development of humoral immunity might also be impaired in the dLN. We therefore immunized in the footpad with nitrophenyl-conjugated (NPconjugated) OVA to assess the capacity of the dLN to mount a T cell-dependent B cell response during both 181/25 and AF15561 infection. Mice were mock infected or infected with 181/25 or AF15561 and 7 days later were immunized with NP-OVA in alum in the left rear footpad. One or 2 weeks later (21-28 days after virus inoculation), we measured the percentage of NP-specific GC B cells in the dLN (Figure 8A). Approximately $70 \%-90 \%$ of the GC B cells present in mock-infected or $181 / 25$-infected NP-OVA-immunized mice were specific for NP at both time points (Figure 8, B-D). In contrast, AF15561-infected mice were unable to mount an NP-specific GC B cell response in the dLN by 7 days after immunization (Figure 8, C and D, and Supplemental Figure 7A). Consistent with these data, the number of NP-specific IgG antibody secreting cells (ASCs) in response to NP-OVA immunization, as measured by ELISPOT, was reduced in AF15561-infected mice compared with mock- or 181/25-infected mice (Supplemental Figure 7B). The NP-specific GC B cell response was partially recovered by 14 days after immunization, suggesting that the dLN remains functionally impaired in AF15561-infected mice out to at least 21 days after inoculation (Figure 8, C and D). These data suggest that the dramatic depletion and mislocalization of lymphocytes in the dLN of AF15561-infected mice impair the ability of B cells to respond to a secondary antigen.

\section{Discussion}

CHIKV causes large outbreaks of rheumatologic disease, resulting in chronic disease symptoms in up to two-thirds of infected individuals $(7,8,15,53)$. The mechanisms by which pathogenic CHIKV strains subvert adaptive immunity and establish persistent infection are poorly understood. We hypothesized that infection with pathogenic or attenuated CHIKV strains results in distinct early events at the site of initiation of the adaptive antiviral response (the $\mathrm{dLN}$ ), and that these differences consequently contribute to CHIKV persistence or clearance in joint tissue. Indeed, we found that acute 181/25 infection resulted in robust dLN enlargement and GC formation, while the dLN of mice infected with AF15561 (or other pathogenic CHIKV), became depleted of lymphocytes and displayed marked fibrosis. Our studies further revealed that lymphocyte depletion following AF15561 infection was not due to a defect in early lymphocyte proliferation, but rather was due to impaired accumulation of naive lymphocytes in the dLN. Reflecting the structural and functional defects in the dLN that occur during pathogenic CHIKV infection, we found that the capacity of the dLN to mount a B cell response to a secondary antigen was severely impaired.

Our observations that persistent, pathogenic CHIKV infection prevents accumulation of naive lymphocytes in the dLN are consistent with recent findings that impaired LN cellularity in aged CHIKVinfected mice correlates with increased viral burden in musculoskeletal tissue (22). Furthermore, reduced leukocyte accumulation and delayed GC formation in the $\mathrm{dLN}$ is associated with increased susceptibility of aged mice to West Nile virus infection (35). Cumulatively, these findings reveal that either virus subversion or immune dysfunction preventing lymphocyte recruitment/accumulation in the dLN have important consequences for viral clearance or susceptibility. Our findings that pathogenic CHIKV markedly disrupts dLN architecture and function expand upon this to suggest a potential downstream mechanism resulting in lymphocyte depletion in the LN that promotes viral persistence.

Three mechanisms account for the majority of lymphocyte accumulation within LNs in response to foreign antigen: increased entry of naive lymphocytes, shutdown of lymphocyte egress, and proliferation of antigen-specific lymphocytes $(37,39,54-58)$. To analyze antigen-specific T cells during CHIKV 
A

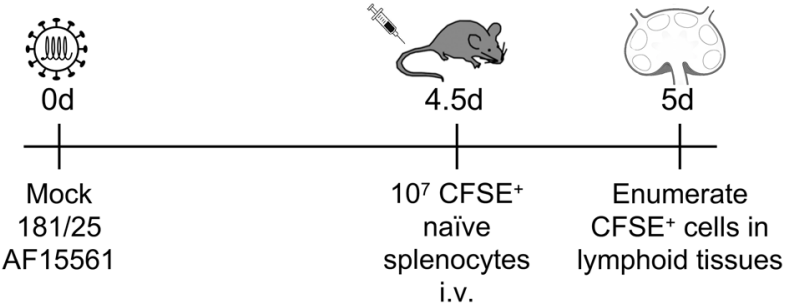

B

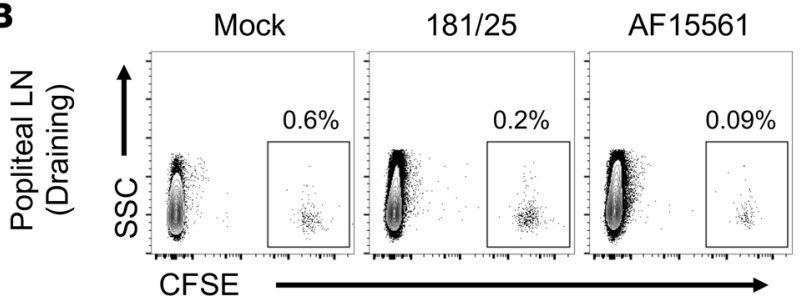

C $\square$ Mock $\square$ 181/25 $\square$ AF15561

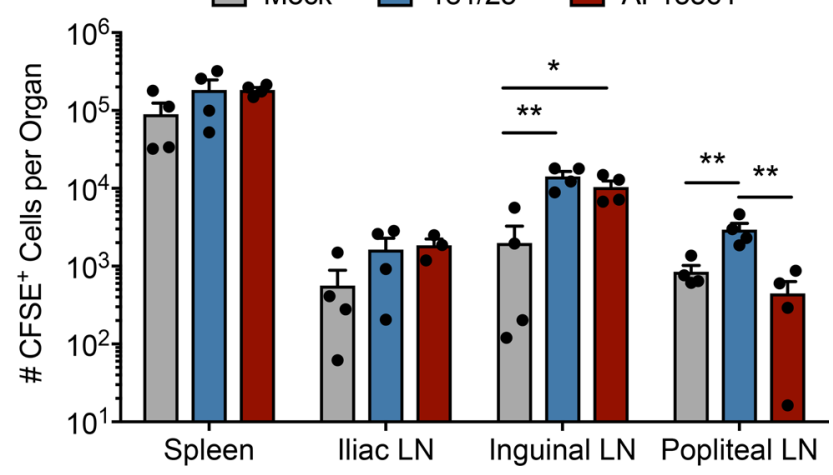

Figure 6. Accumulation of naive lymphocytes in the dLN is impaired during pathogenic CHIKV infection. (A) WT C57BL/6 mice ( $n=4$ per group) were inoculated with $10^{3} \mathrm{PFU}$ of $181 / 25$ or AF15561. At 4.5 dpi, $10^{7}$ naive CFSE-labeled splenocytes were transferred i.v. and enumerated 12 hours later. (B) Flow plots depicting the percentage of CFSE-labeled cells in the dLN. (c) Total number of CFSE-labeled transferred cells per organ (calculated by multiplying the percentage of $\mathrm{CFSE}^{+}$cells by total cells). Data are representative of 2 independent experiments. Each bar represents the mean \pm SEM. ${ }^{*} P<0.05$, ${ }^{*} P<0.01$; one-way ANOVA with Tukey's multiple-comparisons test.

infection, we created new recombinant CHIKV strains expressing commonly used $\mathrm{CD} 4^{+}$and $\mathrm{CD} 8^{+} \mathrm{T}$ cell epitopes from OVA. After infection with these viruses, we did not detect differences in lymphocyte proliferation in the dLN between $181 / 25$ and AF15561 infection either in the entire lymphocyte population or in OVA-specific $\mathrm{CD}^{+}$or $\mathrm{CD} 8^{+} \mathrm{T}$ cells, suggesting that lymphocytes that successfully enter the $\mathrm{dLN}$ receive signals to divide. Instead, our adoptive transfer studies demonstrated that the recruitment of naive lymphocytes into the dLN is impaired during pathogenic CHIKV infection

HEVs are the main portals of entry for naive lymphocytes from the blood to LNs and Peyer's patches (46). The reduced accumulation of naive lymphocytes in the dLN during pathogenic CHIKV infection was associated with decreased HEV cell numbers, decreased HEV area per dLN, and concentration of $\mathrm{CD} 8^{+} \mathrm{T}$ cells on the luminal surface of HEVs, suggesting that poor accumulation of naive lymphocytes in the dLN could be due to a defect in nodal lymphocyte entry. Alterations in expression of the homeostatic chemokines CCL21 and CXCL13 also can disrupt lymphocyte recruitment through HEVs (59-62). While CCL21 levels in the dLN increased early after acutely cleared 181/25 infection, CCL21 did not increase in pathogenic CHIKV-infected dLNs. Though it remains to be established experimentally, this difference in CCL21 expression (either by the HEV themselves or other stromal cells) likely contributes to impaired lymphocyte accumulation in the dLN during AF15561 infection.

Our mapping studies showed that the residues at E2 positions 12 and 82 independently contribute to early lymphocyte depletion in the dLN, as infection with $181 / 25$ containing either or both positions mutated to the WT residue resulted in fewer cells in the dLN than infection with $181 / 25$. Thus, a key finding from our current study - lymphocyte depletion in the $\mathrm{dLN}$ - is linked to amino acid positions in the virus that dictate CHIKV clearance or persistence in musculoskeletal tissue. The residue at E2-82 affects viral titers in SLOs early in infection, as mice infected with viruses with a $\mathrm{G}$ at E2-82 had higher viral titers in the spleen and dLN within the first week of infection $(49,50)$. However, we were unable to detect virus-driven reporter gene expression in LN cells (despite robust expression in cells isolated from the foot) following infection with AF15561.mKate, suggesting that the presence of a $G$ at E2 position 82 does not endow the virus with the 
A

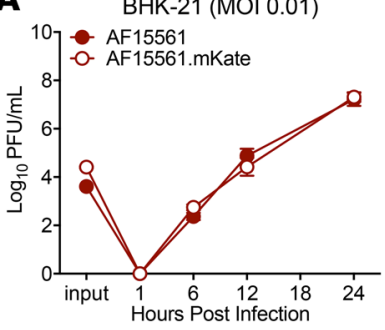

B

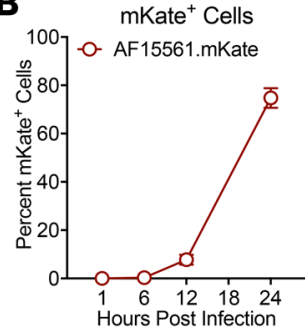

C
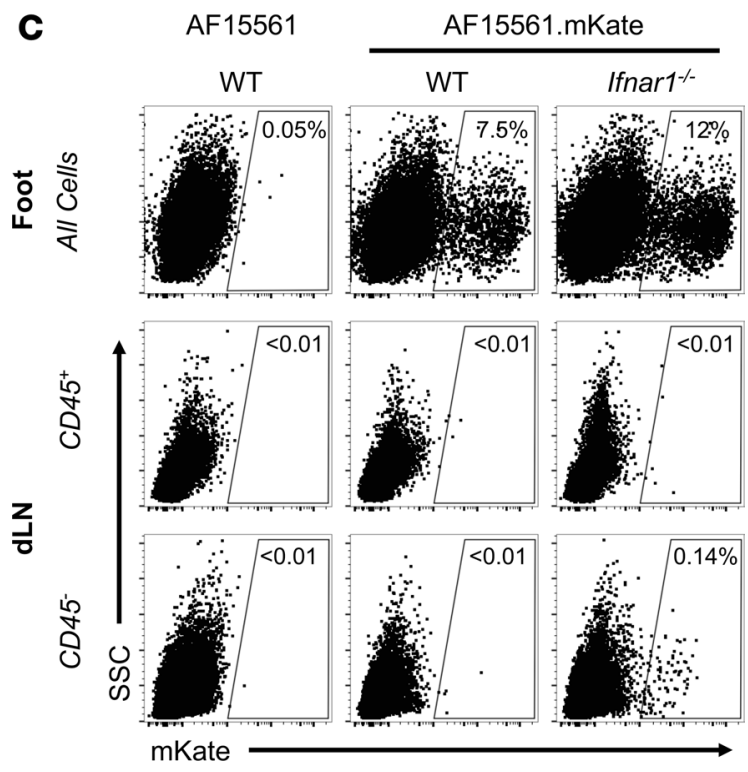

Figure 7. Stromal cells in the dLN are not targets of productive CHIKV infection. (A and B) BHK-21 cells were inoculated with AF15561 or AF15561. mKate and (A) virus yields or (B) the percentage of mKate ${ }^{+}$cells were quantified at the indicated time points. Data represent the mean \pm SEM and are combined from 2 independent experiments. (C) WT or Ifnar1/-- mice were inoculated with $10^{3}$ PFU of AF15561 or AF15561. mKate in both rear footpads. At $24 \mathrm{hpi}$, the foot was processed by collagenase/DNase digest. The percentage of mKate ${ }^{+}$cells was determined by flow cytometry. mKate expression was also determined from CD45+ (top panels) and CD45- (bottom panels) cells from pooled dLNs (4-6 dLNs per group) of WT or Ifnar1//- mice infected with AF15561 or AF15561.mKate for 24 hours. Data are representative of 5 independent experiments.

capacity to productively replicate in LN stromal cells or leukocytes. In addition, LN stromal cells from WT or $I r 3^{-/-}$Irf $^{-1-}$ mice cultured and infected with AF15561 ex vivo did not have detectable viral gene expression or produce infectious virus (data not shown). We did detect a small population of $\mathrm{CD}^{-} 5^{-} \mathrm{mKate}^{+}$cells in the dLN of mice lacking type I IFN signaling, indicating that although LN-resident cells can be infected by AF15561 in the context of type I IFN deficiency, CHIKV does not replicate robustly in the LN even in the absence of a key antiviral defense mechanism. These findings are similar to those of previous studies in which Sindbis virus RNA was detectable in macrophage/DC-like cells in the dLN of Ifnar1 ${ }^{-1-}$ mice, but not WT mice, suggesting that type I IFN suppresses infection of these resident LN cells (63). Thus, the infectious virus and/or viral RNA detected in LNs of CHIKV-infected mice may represent virus that was carried via lymph from peripheral tissue and captured or nonproductive infection rather than bona fide replication in resident LN cells. Therefore, the observed differences in the architecture and function of the dLN during attenuated or pathogenic CHIKV infection appear to be independent of viral replication efficiency in the dLN itself and, instead, are likely due to differential host responses to the 2 infections.

AF15561 infection elicits a poor neutralizing antibody response compared with 181/25 infection (31). The failure of the dLN to accumulate naive lymphocytes, form GCs, and the disrupted follicular organization following AF15561 infection likely contributes to this poorer-quality antibody response. Importantly, our analyses also demonstrate that pathogenic CHIKV infection disrupts the ability of the dLN to generate a humoral response to a secondary antigen. This impaired antibody response may be particularly important for a virus that establishes tissue persistence which should yield continual antigen drainage to the LN. In addition to impaired $\mathrm{B}$ cell responses, we observed fewer endogenous virus-specific $\mathrm{CD} 8^{+} \mathrm{T}$ cells and decreased $\mathrm{CD}^{+} \mathrm{T}$ cell numbers (we did not directly assess endogenous virus-specific $\mathrm{CD} 4^{+} \mathrm{T}$ cells) in the dLN early after AF15561 infection. As each of these cells is important for the generation of a complete adaptive antiviral response, they likely all contribute to impaired virus clearance.

In summary, we reveal that the disruption of dLN architecture and immune responses by pathogenic CHIKV infection represents an additional mechanism by which CHIKV evades adaptive immunity to 
A

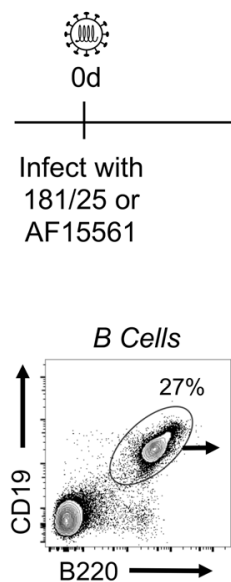

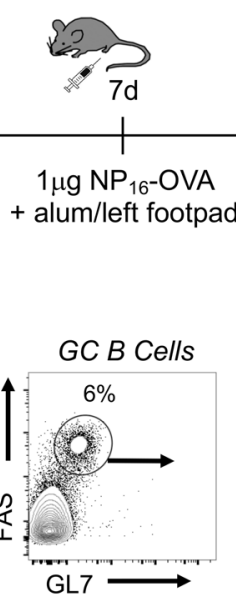
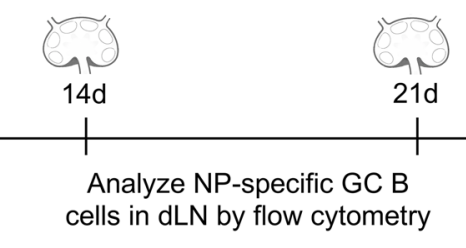

Analyze NP-specific GC B cells in dLN by flow cytometry
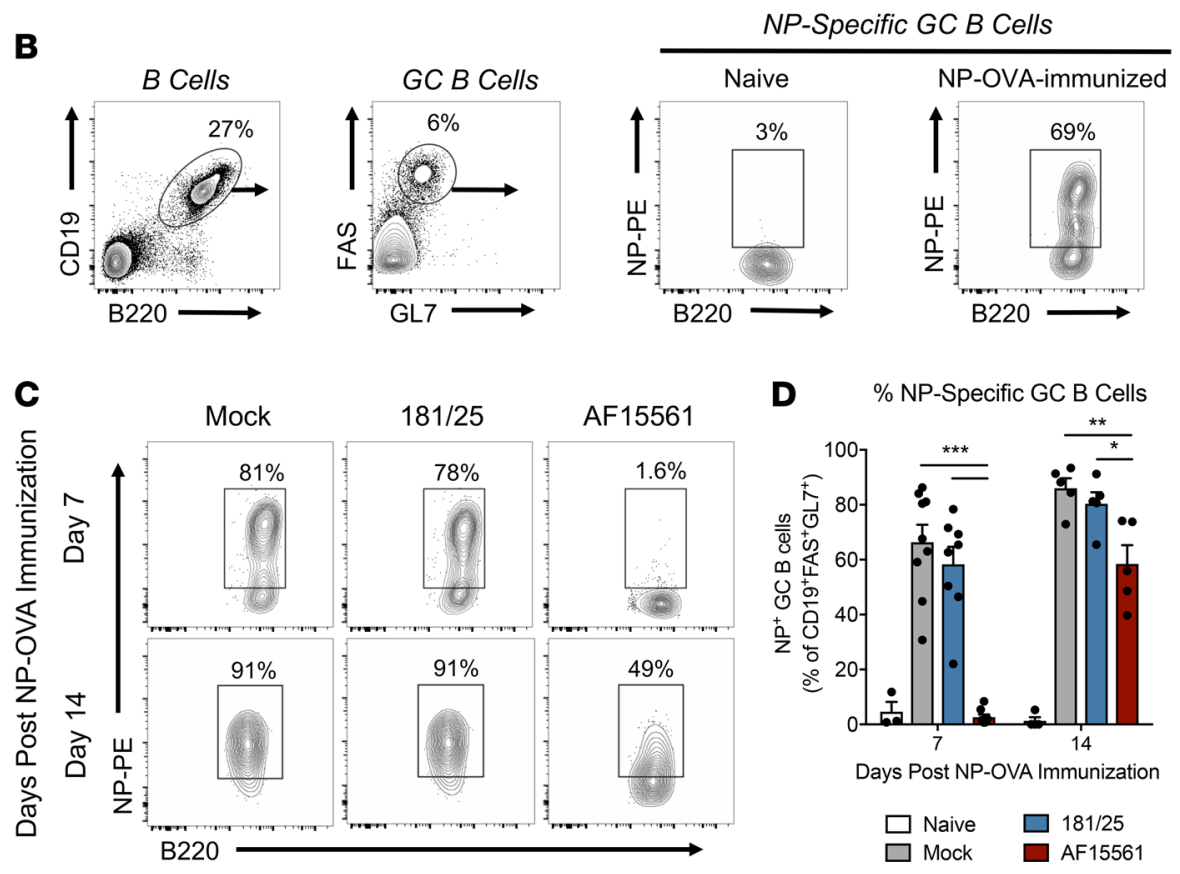

D NP-Specific GC B Cells

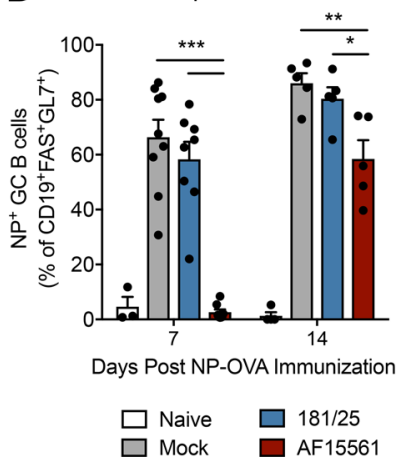

Figure 8. Pathogenic, but not attenuated, CHIKV infection impairs the dLN response to footpad NP-OVA immunization. (A) WT C57BL/6 mice were mock infected or infected with $181 / 25$ or AF15561. At $7 \mathrm{dpi}$, mice were left unimmunized (naive) or immunized with 1 $\mathrm{g} \mathrm{NP}_{16}$-OVA plus alum in the left rear footpad. One or 2 weeks later, the dLN was collected and analyzed for the presence of NP-specific GC B cells by flow cytometry. (B) Gating strategy (after gating on single cells) used to identify NP-specific GC B cells. (C) Flow plots showing percentage of $G C B$ cells binding to NP-PE. (D) Percentage of NP-specific B cells in the dLN at the indicated times after immunization. Data are combined from 2 independent experiments. Each bar represents the mean \pm SEM of 4-5 mice per group. ${ }^{*} P<0.05,{ }^{* *} P$ $<0.01,{ }^{* * *} P<0.001$; one-way ANOVA with Tukey's multiple-comparisons test to compare mock, 181/25, and AF15561 groups (naive not included in the analysis).

establish persistence. Identification of immunological pathways that could be therapeutically blocked or manipulated in order to restore HEV function and lymphocyte recruitment in lymphoid tissues could be beneficial in ameliorating disease symptoms. Understanding what triggers the cascade of events leading to impaired dLN function in order to prevent or reverse virus persistence in musculoskeletal tissue may lead to more effective treatments for chronic CHIKV disease.

\section{Methods}

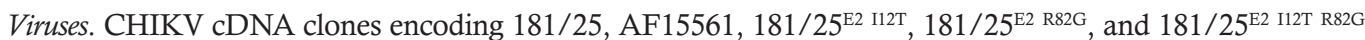
have been described previously (49). The recombinant CHIKV strains expressing the $\mathrm{H}-2 \mathrm{~K}^{\mathrm{b}}$-restricted CD8 OVA $_{257-264}$ epitope (SIINFEKL) and the I-A ${ }^{\mathrm{d}} / \mathrm{I}-\mathrm{A}^{\mathrm{b}}$-restricted CD4 OVA ${ }_{323-339}$ epitope (ISQAVHAAHAEINEA$\mathrm{GR}$ ) in frame with the CHIKV structural polyprotein were generated using a previously described strategy (45).

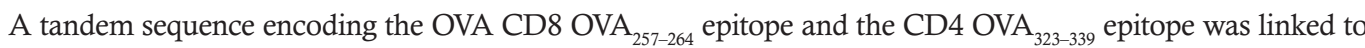
19 amino acids of the 2A protease of foot-and-mouth disease virus (FMDV) and inserted in-frame between capsid and E3 of CHIKV. The AF15561.mKate cDNA construct was made in a similar fashion. Stocks of infectious CHIKV strains were generated from cDNA clones and titered by plaque assay on BHK-21 cells as previously described (44). For some experiments, viral stocks were concentrated by pelleting electroporated supernatants through a $20 \%(\mathrm{w} / \mathrm{v})$ sucrose/PBS cushion at $72,000 \mathrm{~g}$ by ultracentrifugation. Pelleted viruses were resuspended in PBS. All experiments were performed under biosafety level 3 conditions.

Mouse experiments. WT C57BL/6, Ifnar1 ${ }^{-1-}$, and $\mu \mathrm{MT}$ mice were obtained from The Jackson Laboratory, and CD45.1+ OT-II mice were a gift from Claudia Jakubzick (National Jewish Health, Denver, Colorado). For virus infections, mice were used at 3-4 weeks of age. Mice were anesthetized with isoflurane and inoculated with virus in the left rear footpad. For experiments in which HEV cells in the dLN were analyzed, mice were inoculated with virus in both footpads.

For NP-OVA immunizations, mice were immunized with $1 \mu \mathrm{g}$ of $\mathrm{NP}_{16}$-OVA (InvivoGen) in a 1:1 v/v mixture of Alhydrogel adjuvant (InvivoGen) and endotoxin-free $\mathrm{H}_{2} \mathrm{O}$. For adoptive transfer experiments, congenically marked CD45.1 ${ }^{+}$OT-II cells were purified by negative selection using $\mathrm{CD}^{+} \mathrm{T}$ cell isolation 
kits (Stem Cell Technologies). Two hours prior to infection, $10^{5}$ OT-II cells were injected i.v. into C57BL/6 $\left(\mathrm{CD} 45.2^{+}\right)$mice. For short-term adoptive transfers, $10^{7} \mathrm{CFSE}-1$ abeled naive splenocytes from 8-week-old C57BL/ 6 mice were injected i.v. into congenically matched mice 12 hours prior to harvest.

Mice were sacrificed by exposure to isoflurane vapors followed by thoracotomy at the indicated time points. Ankle tissue was dissected and homogenized in TRIzol Reagent (Life Technologies) for RNA analysis with a MagNA Lyser (Roche). The spleen and popliteal LNs were removed and processed for flow cytometry.

Viral RNA analysis. Viral RNA in tissues was quantified by RT-qPCR as previously described (20) using a CHIKV sequence-specific forward primer (5'-TTTGCGTGCCACTCTGG-3'), reverse primer (5'-CGGGTCACCACAAAGTACAA-3'), and probe (5'-ACTTGCTTTGATCGCCTTGGTGAGA-3').

Isolation of cells from lymphoid tissue and flow cytometry. Spleen tissue was processed for flow cytometry as previously described (64). The left popliteal LN was gently homogenized in a Biomasher II tissue homogenizer (Kimble-Chase) in digestion buffer $(2.5 \mathrm{mg} / \mathrm{ml}$ collagenase type I [Worthington Biochemical] $+17 \mu \mathrm{g} / \mathrm{ml}$ DNase I [Roche] in RPMI 1640 [Hyclone]) and incubated for 1 hour at $37^{\circ} \mathrm{C}$ with shaking (130 rpm). For analysis of infected cells in the footpad, foot tissue was homogenized in digestion buffer with glass beads for 1 hour at $37^{\circ} \mathrm{C}$ with shaking $(130 \mathrm{rpm})$. Cells were passed through a $100-\mu \mathrm{m}$ cell strainer (BD Falcon) and total viable cells were determined by hand counting with trypan blue exclusion. For analysis of HEV cells, a pool of 4 popliteal LNs was digested in Liberase/DNase for 1 hour at $37^{\circ} \mathrm{C}$ as previously described (43).

Single-cell suspensions were incubated with anti-mouse FcyRIII/II (2.4G2; BD Pharmingen) for 20 minutes on ice and then stained in FACS staining buffer $(1 \times \mathrm{PBS} / 2 \% \mathrm{FBS})$ for 1 hour on ice with antibodies obtained from BioLegend, BD Biosciences, or eBioscience/ThermoFisher: B220 (RA3-6B2; used to provide continuity between flow cytometric and confocal microscopic analysis), CD4 (RM45), CD8 (53-6.7), TCR $\beta$ (H57-597), CD3 (145-2C11), Ki67 (16A8), CD45.1 (A20), CD45 (30-F11), CD31 (390), PNAd (MECA-79), CD19 (6D5), FAS (15A7), GL7 (GL7), CXCR5 (L138D7), PD-1 (J43), NK1.1 (PK136), ICAM-1 (YN1/1.7.4). PE-labeled H-2K b SIINFEKL tetramer was kindly provided by Ross Kedl (University of Colorado Anschutz Medical Campus, Aurora, Colorado, USA). NP ${ }_{40} \mathrm{PE}$ was obtained from Biosearch Technologies. For intracellular analysis of Ki67 expression, cells were processed as previously described (64). Cells were fixed overnight in $1 \times$ PBS/1\% paraformaldehyde (PFA) and analyzed on a BD LSR Fortessa cytometer using FACSDiva software. Further analysis was performed using FlowJo software (Tree Star).

For determination of live, dead, or apoptotic lymphocytes, cells were washed once with PBS, incubated with LIVE/DEAD Fixable Aqua Dead Cell Stain (AquaVI; ThermoFisher) for 15 minutes at room temperature (RT), and then washed twice with PBS prior to surface staining. After surface staining, cells were incubated with Annexin V diluted in Annexin V Binding Buffer (BioLegend) for 15 minutes at RT, washed once in binding buffer, and resuspended in binding buffer/1\% PFA for acquisition.

CCL21 ELISA. LNs were collected at 1 or 3 dpi and placed in $0.5 \mathrm{ml} \mathrm{PBS/1 \%} \mathrm{BSA} \mathrm{(w/v).} \mathrm{LNs} \mathrm{were}$ homogenized in a Biomasher II tissue homogenizer and CCL21 was measured using an ELISA kit from R\&D Systems.

Histology. LNs were fixed in 4\% PFA and embedded in paraffin. Five-micrometer sections were stained with H\&E. Digital images were obtained using a Nikon Eclipse Ti-S microscope and NIS-Elements AR software. Microscopic lesions were graded using a 4-point scale: 1, minimal; 2, mild; 3, moderate; and 4, marked; as compared with at least 5 uninfected control animals. Fibrosis was diagnosed when there was replacement of lymphocytes with fibroblasts and excess fibrous connective tissue. Scoring of GCs was performed using a standard severity 4-point scale: 1, minimal; 2, mild; 3, moderate; and 4, marked; as compared with at least 5 uninfected control animals. If none were present, then a score of 0 was assigned. Due to normal variation in size of LNs (and therefore normal variation in number of follicles), determining the severity grade provides a more accurate assessment of GCs compared with simply counting GCs. All histological evaluations were done using H\&E-stained slides by an ACVP board-certified pathologist.

Immunofluoresence and confocal microscopy. LNs were fixed in $0.5 \mathrm{ml}$ of phosphate buffer containing $0.1 \mathrm{M}$ L-lysine, $2 \% \mathrm{PFA}$, and $2.1 \mathrm{mg} / \mathrm{ml} \mathrm{NaIO}_{4}$ at $\mathrm{pH} 7.4$ for 24 hours at $4^{\circ} \mathrm{C}$, followed by incubation in $30 \%$ sucrose phosphate-buffered solution for 48 hours, then in 30\% sucrose/PBS for 24 hours. Nodes were then embedded in optimal-cutting-temperature medium (Electron Microscopy Sciences) and frozen in dry-ice-cooled isopentane. 
Eighteen-micrometer sections were cut on a Leica cryostat (Leica Microsystems). Sections were blocked with $5 \%$ goat, donkey, bovine, rat, or rabbit serum and then stained with one or more of the following: B220 (clone RA3-6B2, ThermoFisher), CD8 $\alpha$ (clone 53-6.7, ThermoFisher), ERTR-7 (rat monoclonal, BioXCell) and/or PNAd (MECA-79, BioXCell). Sections were incubated with anti-rat IgM Alexa 647 to detect PNAd, or alone as needed for controls, and images were acquired using identical PMT (photomultiplier tube) and laser power settings on a Leica SP5 confocal equipped with HyD detectors (Leica). Confocal images were obtained of the entire popliteal LN (representing approximately a 7- $\mathrm{mm}^{2}$ imaged area) and individual fields (tiles) were merged into a single image file. Images were analyzed using Imaris v9.02 software (Bitplane).

For qualitative analyses of HEVs per section and area per HEV, the Surfaces function of Imaris was used to perform isosurface visualization of the PNAd channel (Supplemental Figure 5F). After surfaces representing each individual $\mathrm{HEV}$ were created and manually inspected for accuracy, the area for each individual surface (HEV), the summed area of all surfaces per LN section (total HEV area/section), and the number of surfaces (HEVs/section) were exported directly from the isosurface calculation of Imaris. For consistency, LNs from different infections were stained in a single batch and confocal images collected under identical acquisition conditions.

To calculate the concentration coefficient of the $\mathrm{CD}^{+}$fluorescence signal with the HEV lumen, the mean fluorescence signal of CD8 within HEVs was divided by the mean fluorescence signal of CD8 on the entire LN section (in order to control for the increased number of $\mathrm{CD}^{+} \mathrm{T}$ cells in the $\mathrm{dLN}$ after $181 / 25$ infection compared with AF15561 infection).

NP ELISPOT. For NP-specific IgG enzyme-linked immunospot assay (ELISPOT), popliteal LNs were removed at 7 days after NP-OVA immunization and processed into a single-cell suspension as above. Two-fold dilutions of cells (starting at $2.5 \times 10^{5}$ ) were plated on filter plates (EMP Millipore MultiScreen HTS-IP) coated with $10 \mu \mathrm{g} / \mathrm{mL} \mathrm{NP}_{20}$-BSA (Biosearch Technologies) and incubated for 5 hours at $37^{\circ} \mathrm{C}$. Biotin-conjugated anti-IgG and streptavidin-conjugated horseradish peroxidase (HRP) (both from Southern Biotech) were used for detection. For development, $200 \mu 1$ 3-amino-9-ethylcarbazole (AEC) solution (Sigma-Aldrich) was added to $9 \mathrm{ml} 0.1 \mathrm{M}$ sodium acetate buffer containing $4 \mu 130 \% \mathrm{H}_{2} \mathrm{O}_{2}$. Spots were analyzed on a C.T.L. ELISPOT Reader using ImmunoSpot 5.1 software (Cellular Technology).

Statistics. Statistical analysis was conducted using GraphPad Prism 6.0. $P$ values less than 0.05 were considered statistically significant $\left({ }^{*} P<0.05,{ }^{* *} P<0.01,{ }^{* * *} P<0.001\right)$.

Study approval. All animal work was approved by the Institutional Animal Care and Use Committee at the University of Colorado School of Medicine (Assurance Number A3269-01).

\section{Author contributions}

MKM and TEM conceptualized the study. MKM, BJD, GVR, EDL, NAM, SAE, and HDH performed the investigation. BAT, $\mathrm{HDH}$, and TEM provided resources. MKM and TEM wrote the original draft of the manuscript. MKM, BAT, HDH, and TEM reviewed and edited the manuscript. MKM, SAE, BAT, HDH, and TEM acquired funding. BAT, HDH, and TEM supervised the study.

\section{Acknowledgments}

This work was supported by Public Health Service grants U19 AI109680 and R01 AI108725 (to T.E. Morrison), R01AI121209 (to B.A. Tamburini), and F32 AI122463 (to M.K. McCarthy) from the National Institute of Allergy and Infectious Diseases (NIAID), NIH. H.D. Hickman is supported by the Intramural Research Program of the NIAID, NIH. This research was also supported in part by the Intramural Research Program of the NIH, National Institute of Environmental Health Sciences (NIEHS) (to S.A. Elmore). We thank David Heinz at the National Jewish Pathology Core and Erin Kitten at the University of Colorado School of Medicine Flow Cytometry Shared Resource facility for assistance with histology and flow cytometry, respectively. The authors thank Terence S. Dermody and Linda van Dyk for critical reading of the manuscript.

Address correspondence to: Thomas E. Morrison, Associate Professor, Department of Immunology and Microbiology, University of Colorado School of Medicine, 12800 East 19th Avenue, Mail Stop 8333, Aurora, Colorado 80045, USA. Phone: 303.724.4283; Email: thomas.morrison@ucdenver.edu. 
1. AbuBakar S, Sam IC, Wong PF, MatRahim N, Hooi PS, Roslan N. Reemergence of endemic Chikungunya, Malaysia. Emerging Infect Dis. 2007;13(1):147-149.

2. Depoortere E, Salmaso S, Pompa MG, Guglielmetti P, Coulombier D. Chikungunya in Europe. Lancet. 2008;371(9614):723.

3. Josseran L, et al. Chikungunya disease outbreak, Reunion Island. Emerging Infect Dis. 2006;12(12):1994-1995.

4. Kaur P, et al. Chikungunya outbreak, South India, 2006. Emerging Infect Dis. 2008;14(10):1623-1625.

5. Leo YS, Chow AL, Tan LK, Lye DC, Lin L, Ng LC. Chikungunya outbreak, Singapore, 2008. Emerging Infect Dis. 2009;15(5):836-837.

6. Olowokure B, et al. The Caribbean response to chikungunya. Lancet Infect Dis. 2014;14(11):1039-1040.

7. Borgherini G, et al. Persistent arthralgia associated with chikungunya virus: a study of 88 adult patients on reunion island. Clin Infect Dis. 2008;47(4):469-475.

8. Schilte C, et al. Chikungunya virus-associated long-term arthralgia: a 36-month prospective longitudinal study. PLoS Negl Trop Dis. 2013;7(3):e2137.

9. Powers AM, Logue $\mathrm{CH}$. Changing patterns of chikungunya virus: re-emergence of a zoonotic arbovirus. J Gen Virol. 2007;88(Pt 9):2363-2377.

10. Larrieu S, et al. Factors associated with persistence of arthralgia among Chikungunya virus-infected travellers: report of 42 French cases. J Clin Virol. 2010;47(1):85-88.

11. Murillo-Zamora E, Mendoza-Cano O, Trujillo-Hernández B, Alberto Sánchez-Piña R, Guzmán-Esquivel J. Persistent arthralgia and related risks factors in laboratory-confirmed cases of Chikungunya virus infection in Mexico. Rev Panam Salud Publica. 2017;41:e72.

12. Parola P, Simon F, Oliver M. Tenosynovitis and vascular disorders associated with Chikungunya virus-related rheumatism. Clin Infect Dis. 2007;45(6):801-802.

13. Simon F, et al. Chikungunya infection: an emerging rheumatism among travelers returned from Indian Ocean islands. Report of 47 cases. Medicine (Baltimore). 2007;86(3):123-137.

14. Sissoko D, et al. Post-epidemic Chikungunya disease on Reunion Island: course of rheumatic manifestations and associated factors over a 15-month period. PLoS Negl Trop Dis. 2009;3(3):e389.

15. Gérardin P, et al. Predictors of Chikungunya rheumatism: a prognostic survey ancillary to the TELECHIK cohort study. Arthritis Res Ther. 2013;15(1):R9.

16. Ozden S, et al. Human muscle satellite cells as targets of Chikungunya virus infection. PLoS ONE. 2007;2(6):e527.

17. Hoarau JJ, et al. Persistent chronic inflammation and infection by Chikungunya arthritogenic alphavirus in spite of a robust host immune response. J Immunol. 2010;184(10):5914-5927.

18. Labadie $\mathrm{K}$, et al. Chikungunya disease in nonhuman primates involves long-term viral persistence in macrophages. J Clin Invest. 2010;120(3):894-906.

19. Messaoudi I, et al. Chikungunya virus infection results in higher and persistent viral replication in aged rhesus macaques due to defects in anti-viral immunity. PLoS Negl Trop Dis. 2013;7(7):e2343.

20. Hawman DW, et al. Chronic joint disease caused by persistent Chikungunya virus infection is controlled by the adaptive immune response. J Virol. 2013;87(24):13878-13888.

21. Poo YS, et al. Multiple immune factors are involved in controlling acute and chronic chikungunya virus infection. PLoS Negl Trop Dis. 2014;8(12):e3354.

22. Uhrlaub JL, et al. Dysregulated TGF- $\beta$ production underlies the age-related vulnerability to Chikungunya virus. PLoS Pathog. 2016;12(10):e1005891.

23. Teo TH, et al. A pathogenic role for $\mathrm{CD}^{+} \mathrm{T}$ cells during Chikungunya virus infection in mice. J Immunol. 2013;190(1):259-269.

24. Schwartz O, Albert ML. Biology and pathogenesis of chikungunya virus. Nat Rev Microbiol. 2010;8(7):491-500.

25. MacDonald GH, Johnston RE. Role of dendritic cell targeting in Venezuelan equine encephalitis virus pathogenesis. $J$ Virol. 2000;74(2):914-922.

26. Hickman $\mathrm{HD}$, et al. Direct priming of antiviral CD8 ${ }^{+} \mathrm{T}$ cells in the peripheral interfollicular region of lymph nodes. Nat Immunol. 2008;9(2):155-165.

27. Junt T, et al. Subcapsular sinus macrophages in lymph nodes clear lymph-borne viruses and present them to antiviral B cells. Nature. 2007;450(7166):110-114.

28. Karrer U, et al. On the key role of secondary lymphoid organs in antiviral immune responses studied in alymphoplastic (aly/ aly) and spleenless (Hox11(-)/-) mutant mice. J Exp Med. 1997;185(12):2157-2170.

29. Levitt NH, Ramsburg HH, Hasty SE, Repik PM, Cole FE, Lupton HW. Development of an attenuated strain of chikungunya virus for use in vaccine production. Vaccine. 1986;4(3):157-162.

30. Gorchakov R, et al. Attenuation of Chikungunya virus vaccine strain 181/clone 25 is determined by two amino acid substitutions in the E2 envelope glycoprotein. J Virol. 2012;86(11):6084-6096.

31. Hawman DW, et al. Pathogenic Chikungunya virus evades B cell responses to establish persistence. Cell Rep. 2016;16(5):1326-1338

32. Klonowski KD, Marzo AL, Williams KJ, Lee SJ, Pham QM, Lefrançois L. CD8 T cell recall responses are regulated by the tissue tropism of the memory cell and pathogen. J Immunol. 2006;177(10):6738-6746.

33. Suresh M, et al. Role of lymphotoxin alpha in T-cell responses during an acute viral infection. J Virol. 2002;76(8):3943-3951.

34. Olson MR, McDermott DS, Varga SM. The initial draining lymph node primes the bulk of the CD8 T cell response and influences memory T cell trafficking after a systemic viral infection. PLoS Pathog. 2012;8(12):e1003054.

35. Richner JM, et al. Age-dependent cell trafficking defects in draining lymph nodes impair adaptive immunity and control of West Nile virus infection. PLoS Pathog. 2015;11(7):e1005027.

36. Victora GD, Nussenzweig MC. Germinal centers. Annu Rev Immunol. 2012;30:429-457.

37. Shiow LR, et al. CD69 acts downstream of interferon-alpha/beta to inhibit S1P1 and lymphocyte egress from lymphoid organs. Nature. 2006;440(7083):540-544

38. Schulz O, et al. Hypertrophy of infected Peyer's patches arises from global, interferon-receptor, and CD69-independent shutdown of lymphocyte egress. Mucosal Immunol. 2014;7(4):892-904.

39. Kunz S, Oberle K, Sander A, Bogdan C, Schleicher U. Lymphadenopathy in a novel mouse model of Bartonella-induced cat 
scratch disease results from lymphocyte immigration and proliferation and is regulated by interferon-alpha/beta. Am J Pathol. 2008;172(4):1005-1018.

40. Acton SE, et al. Dendritic cells control fibroblastic reticular network tension and lymph node expansion. Nature. 2014;514(7523):498-502.

41. Angeli V, et al. B cell-driven lymphangiogenesis in inflamed lymph nodes enhances dendritic cell mobilization. Immunity. 2006;24(2):203-215.

42. Chyou S, et al. Coordinated regulation of lymph node vascular-stromal growth first by CD11 $\mathrm{c}^{+}$cells and then by $\mathrm{T}$ and $\mathrm{B}$ cells. J Immunol. 2011;187(11):5558-5567.

43. Tamburini BA, Burchill MA, Kedl RM. Antigen capture and archiving by lymphatic endothelial cells following vaccination or viral infection. Nat Commun. 2014;5:3989.

44. Morrison TE, et al. A mouse model of chikungunya virus-induced musculoskeletal inflammatory disease: evidence of arthritis, tenosynovitis, myositis, and persistence. Am J Pathol. 2011;178(1):32-40.

45. Burrack KS, Montgomery SA, Homann D, Morrison TE. CD8 ${ }^{+}$T cells control Ross River virus infection in musculoskeletal tissues of infected mice. J Immunol. 2015;194(2):678-689.

46. Girard JP, Moussion C, Förster R. HEVs, lymphatics and homeostatic immune cell trafficking in lymph nodes. Nat Rev Immunol. 2012;12(11):762-773.

47. Berg EL, Robinson MK, Warnock RA, Butcher EC. The human peripheral lymph node vascular addressin is a ligand for LECAM-1, the peripheral lymph node homing receptor. J Cell Biol. 1991;114(2):343-349.

48. Streeter PR, Rouse BT, Butcher EC. Immunohistologic and functional characterization of a vascular addressin involved in lymphocyte homing into peripheral lymph nodes. J Cell Biol. 1988;107(5):1853-1862.

49. Ashbrook AW, et al. Residue 82 of the Chikungunya virus E2 attachment protein modulates viral dissemination and arthritis in mice. J Virol. 2014;88(21):12180-12192.

50. Gardner CL, Burke CW, Higgs ST, Klimstra WB, Ryman KD. Interferon-alpha/beta deficiency greatly exacerbates arthritogenic disease in mice infected with wild-type chikungunya virus but not with the cell culture-adapted live-attenuated $181 / 25$ vaccine candidate. Virology. 2012;425(2):103-112.

51. Rudd PA, et al. Interferon response factors 3 and 7 protect against Chikungunya virus hemorrhagic fever and shock. $J$ Virol. 2012;86(18):9888-9898.

52. Schilte C, Buckwalter MR, Laird ME, Diamond MS, Schwartz O, Albert ML. Cutting edge: independent roles for IRF-3 and IRF-7 in hematopoietic and nonhematopoietic cells during host response to Chikungunya infection. J Immunol. 2012;188(7):2967-2971.

53. Gérardin P, et al. Chikungunya virus-associated encephalitis: A cohort study on La Réunion Island, 2005-2009. Neurology 2016;86(1):94-102.

54. Carvalho LP, et al. Lymph node hypertrophy following Leishmania major infection is dependent on TLR9. J Immunol. 2012;188(3):1394-1401.

55. MartIn-Fontecha A, et al. Regulation of dendritic cell migration to the draining lymph node: impact on T lymphocyte traffic and priming. J Exp Med. 2003;198(4):615-621.

56. McConnell I, Hopkins J. Lymphocyte traffic through antigen-stimulated lymph nodes. I. Complement activation within lymph nodes initiates cell shutdown. Immunology. 1981;42(2):217-223.

57. McLachlan JB, et al. Mast cell-derived tumor necrosis factor induces hypertrophy of draining lymph nodes during infection. Nat Immunol. 2003;4(12):1199-1205.

58. Schwab SR, Cyster JG. Finding a way out: lymphocyte egress from lymphoid organs. Nat Immunol. 2007;8(12):1295-1301.

59. St John AL, Abraham SN. Salmonella disrupts lymph node architecture by TLR4-mediated suppression of homeostatic chemokines. Nat Med. 2009;15(11):1259-1265.

60. Mueller SN, et al. Regulation of homeostatic chemokine expression and cell trafficking during immune responses. Science. 2007;317(5838):670-674.

61. Benedict CA, De Trez C, Schneider K, Ha S, Patterson G, Ware CF. Specific remodeling of splenic architecture by cytomegalovirus. PLoS Pathog. 2006;2(3):e16.

62. Soudja SM, et al. Disrupted lymph node and splenic stroma in mice with induced inflammatory melanomas is associated with impaired recruitment of $\mathrm{T}$ and dendritic cells. PLoS ONE. 2011;6(7):e22639.

63. Ryman KD, Klimstra WB, Nguyen KB, Biron CA, Johnston RE. Alpha/beta interferon protects adult mice from fatal Sindbis virus infection and is an important determinant of cell and tissue tropism. $J$ Virol. 2000;74(7):3366-3378.

64. Burrack KS, et al. Myeloid cell Arg1 inhibits control of arthritogenic alphavirus infection by suppressing antiviral T cells. $P L o S$ Pathog. 2015;11(10):e1005191. 